\title{
In silico selection of functionally important proteins from the mialome of Ornithodoros erraticus ticks and assessment of their protective efficacy as vaccine targets
}

\author{
Ricardo Pérez-Sánchez ${ }^{1 *}\left(\mathbb{0}\right.$, Raúl Manzano-Román ${ }^{1,2}$, Prosper Obolo-Mvoulouga ${ }^{1}$ and Ana Oleaga ${ }^{1}$
}

\begin{abstract}
Background: New candidate protective antigens for tick vaccine development may be identified by selecting and testing antigen candidates that play key biological functions. After blood-feeding, tick midgut overexpresses proteins that play essential functions in tick survival and disease transmission. Herein, Ornithodoros erraticus midgut transcriptomic and proteomic data were examined in order to select functionally significant antigens upregulated after feeding to be tested as vaccine candidate antigens.

Methods: Transcripts annotated as chitinases, tetraspanins, ribosomal protein P0 and secreted proteins/peptides were mined from the recently published $O$. erraticus midgut transcriptome and filtered in a second selection step using criteria based on upregulation after feeding, predicted antigenicity and expression in the midgut proteome. Five theoretical candidate antigens were selected, obtained as recombinant proteins and used to immunise rabbits: one chitinase (CHI), two tetraspanins (TSPs), the ribosomal protein PO (RPPO) and one secreted protein PK-4 (PK4).

Results: Rabbit vaccination with individual recombinant candidates induced strong humoral responses that mainly reduced nymph moulting and female reproduction, providing 30.2\% (CHI), 56\% (TSPs), 57.5\% (RPP0) and 57.8\% (PK4) protection to O. erraticus infestations and 19.6\% (CHI), 11.1\% (TSPs), 0\% (RPP0) and 8.1\% (PK4) cross-protection to infestations by the African tick Ornithodoros moubata. The joint vaccine efficacy of the candidates was assessed in a second vaccine trial reaching $66.3 \%$ protection to 0 . erraticus and $25.6 \%$ cross-protection to O. moubata.

Conclusions: These results (i) indicate that argasid chitinases and RPPO are promising protective antigens, as has already been demonstrated for ixodid chitinases and RPPO, and could be included in vaccines targeting multiple tick species; (ii) reveal novel protective antigens tetraspanins and secreted protein PK-4, never tested before as protective antigens in ticks; and (iii) demonstrate that multi-antigenic vaccines increased vaccine efficacy compared with individual antigens. Lastly, our data emphasize the value of the tick midgut as a source of protective candidate antigens in argasids for tick control.
\end{abstract}

Keywords: Ornithodoros erraticus, Mialome, Vaccines, Chitinase, Ribosomal protein P0, Tetraspanin, Secreted protein PK-4

\footnotetext{
*Correspondence: ricardo.perez@irnasa.csic.es

1 Parasitología Animal, Instituto de Recursos Naturales y Agrobiología de Salamanca (IRNASA, CSIC), Cordel de Merinas, 40-52, 37008 Salamanca, Spain

Full list of author information is available at the end of the article
} (http://creativecommons.org/licenses/by/4.0/), which permits unrestricted use, distribution, and reproduction in any medium, provided you give appropriate credit to the original author(s) and the source, provide a link to the Creative Commons license, and indicate if changes were made. The Creative Commons Public Domain Dedication waiver (http://creativecommons.org/ publicdomain/zero/1.0/) applies to the data made available in this article, unless otherwise stated. 


\section{Background}

Tick infestations and tick-borne diseases are a growing threat for human and animal health globally [1]. Ornithodoros erraticus is an argasid tick species reported to be the main vector of tick-borne human relapsing fever (TBRF) and of African swine fever (ASF) in the Mediterranean Basin [2-4]. Moreover, O. erraticus is the typespecies of the "O. erraticus complex", and several species in this complex, including O. asperus, O. lahorensis, $O$. tartakovsky and O. tholozani, are distributed through the Middle East, the Caucasus, the Russian Federation and the Far East, where they transmit different species of TBRF-causing borreliae [5-7] and where the ASF virus has penetrated and spread out of control in the last decade [8-11]. Although this has not been experimentally proven hitherto, if these tick species in the "O. erraticus complex" were also competent vectors of the ASF virus, their presence in anthropic environments would significantly increase the transmission and long-term persistence of ASF in this wide region. Accordingly, the effective prevention and control of TBRF and ASF would necessarily go through eradicating the Ornithodoros vectors from at least anthropic environments [12]. While chemical acaricide agents are not effective against these Ornithodoros ticks [12], alternative methods for the control of ticks are urgently required and tick vaccines have been validated as an effective sustainable method for the control of tick infestations and tick-borne diseases [13-15].

The tick midgut is an essential organ for tick survival because it manages host blood digestion and the absorption of the released nutrients, contributes to protection against host immunity and participates in blood-borne pathogen infection and transmission [16-19]. Accordingly, midgut proteins involved in these processes may be interesting targets for developing vaccines aimed to control ticks and tick-borne pathogens. In fact, in our previous studies with Ornithodoros ticks we have observed that plasma membrane-associated proteins from tick enterocytes induce protective immune responses in vaccinated animals [20-22].

The midgut transcriptomes and proteomes (mialomes) of O. erraticus females, taken before feeding and 48 hours post-feeding, have recently been obtained providing a wealth of information on the physiology of blood digestion and the functionally relevant proteins that are upregulated in the $O$. erraticus midgut in response to blood feeding (BioProject PRJNA377416) [23, 24].

Some of these proteins, including two aquaporins, one ATP-binding cassette $(\mathrm{ABC})$ transporter and one selenoprotein $\mathrm{T}$ (OeSEL), have recently been selected and tested as protective candidate antigens, obtaining partial protection against $O$. erraticus infestations, which reached $47.5 \%$ vaccine efficacy for OeSEL [25]. Thus, new candidate protective antigens from Ornithodoros ticks are still needed, which may be identified by searching the O. erraticus mialome for proteins playing a role in physiological processes that are essential for tick survival.

Accordingly, in the present study, we have focused on midgut chitinases, tetraspanins and the $60 \mathrm{~S}$ acidic ribosomal protein P0 (RPP0) for the selection and testing of new candidate protective antigens.

Chitinases are enzymes that hydrolyse the $\beta-1,4-$ glycosidic bond between $\mathrm{N}$-acetyl-D-glucosamine moieties, mainly found in chitin. In arthropods, chitin is a major structural component of the exoskeleton and an essential part of the peritrophic matrix lining the gut epithelium. The peritrophic matrix is a permeability barrier that protects the midgut from mechanical damage, toxins and pathogens. In order to grow and develop, arthropods need to remodel their chitin-containing structures, which requires the involvement of chitinases and chitin synthases [26]. Arthropod chitinases belong to the glycoside hydrolase-18 (GH18) family, which includes numerous and diverse enzymes with a modular structure composed of a combination of catalytic domains (GH18 domains), cysteine-rich chitin-binding domains, and serine/threonine-rich linker domains. These chitinases are differently expressed between developmental stages and tissues and play distinct functions [27]. In ticks, chitinases have been found in salivary glands, the midgut, ovaries, Malpighian tubules, synganglion and the epidermis, where they contribute to attaching and feeding, degradation of the chitinous endocuticle during moulting, and regulation of the turnover and porosity of the chitin-containing peritrophic matrix [24, 28-30]. Accordingly, tick chitinases have been considered as potential bioacaricides and vaccine targets for tick control [28, 31]. More recently, RNAi gene knockdown of a chitinase of Amblyomma americanum was shown to reduce female feeding performance and fecundity [29].

Tetraspanins are evolutionarily conserved membrane proteins that tend to associate with one another and to cluster dynamically with numerous and varied partner proteins forming tetraspanin-enriched microdomains in the cell membrane. Thus, tetraspanins are involved in the coordination of numerous intracellular and intercellular biological processes including signalling, cell proliferation, adhesion and migration, cell fusion, immune defence and host-parasite interactions [3234]. Increasing evidence shows that host tetraspanins (vertebrate and invertebrate) are exploited by viruses, bacteria, protozoa and pathogenic fungi for infection, dissemination and transmission [35-39]. Conversely, endogenous tetraspanins of lower parasitic eukaryotes, including schistosomes and filarial nematodes, 
are often pivotal for infectivity and survival in their hosts [33]. Due to their roles in pathogen-vector-host interactions, tetraspanins are potential targets for new therapies and vaccines aimed at controlling parasite infections, arthropod vectors and vector-borne diseases. Actually, a tetraspanin-based candidate vaccine to prevent human schistosomiasis is currently under development [33, 40]. Regarding ticks, tetraspanins might also be suitable targets for vaccines aimed at controlling tick infestations and tick-transmitted pathogens, but no studies on the protective efficacy of tick tetraspanins have been hitherto undertaken.

The $60 \mathrm{~S}$ acidic ribosomal protein P0 (RPP0) is a highly conserved multifunctional protein in eukaryotes. It is a structural component of ribosomes involved in protein synthesis and its absence results in defective $60 S$ ribosomal subunits, which are inactive for protein synthesis, and cell death [41-43]. Additionally, a regulatory role of RPP0 in DNA repair, apoptosis, cell development and carcinogenesis has also been documented [44]. RPP0 has also been shown to associate to membrane proteins on the cell surface of yeast, apicomplexan parasites and mammalian cell lines, but its function there still is unknown [45]. RPP0 has proven to be immunogenic and a promising vaccine candidate against several parasitic protozoans [42], and it has also been explored as a protective candidate antigen in ixodid ticks. RNAi gene knockdown of RPP0 in Haemaphysalis longicornis resulted in a dramatic reduction in tick feeding and a mortality of $96 \%$, suggesting that RPPO is essential for blood ingestion and tick viability [41]. More recently, rabbit vaccination with a synthetic peptide from the RPPO of Rhipicephalus sanguineus showed $90 \%$ vaccine efficacy against this species, and cattle vaccination with this same peptide provided $96 \%$ vaccine efficacy against Rhipicephalus microplus infestations [42, 43].

Besides these proteins, we were also interested in a group of transcripts from the O. erraticus mialome annotated as "secreted protein" and "secreted peptide". Despite most of them have unknown functions, they may be interesting as candidate antigens because secreted antigens are easily reached by host antibodies in the tick midgut lumen.

All the above-mentioned observations highlight these tick proteins as potential protective antigens and prompted us to study their vaccine efficacy. Thus, in the present study, one chitinase, two tetraspanins, the ribosomal protein $\mathrm{P} 0$ and one secreted protein $\mathrm{PK}-4$ were selected from the $O$. erraticus mialome, and their individual and joint vaccine efficacy tested to O. erraticus and the African Ornithodoros moubata soft ticks.

\section{Methods}

\section{Ticks and tick material}

The O. erraticus and O. moubata ticks used in this study came from the laboratory colonies kept at IRNASA (CSIC), Spain. The O. erraticus colony originated from specimens captured in Sanchón de la Ribera $\left(41^{\circ} 5^{\prime} 0^{\prime \prime} \mathrm{N}\right.$, $\left.6^{\circ} 25^{\prime} 0^{\prime \prime} \mathrm{W}\right)$, Salamanca Province, western Spain. The O. moubata colony was started from specimens kindly provided by the Institute for Animal Health in Pirbright (Surrey, UK). The ticks are regularly fed on rabbits (New Zealand white) and maintained at $28{ }^{\circ} \mathrm{C}, 85 \%$ relative humidity and a 12:12 h L:D photoperiod.

Midguts from engorged $O$. erraticus females $48 \mathrm{~h}$ postfeeding (hpf) were obtained as described by [24] and preserved in RNA-later (Ambion, Austin, USA) for RNA extraction. Total RNA was extracted and purified using the RNeasy Mini Kit (Qiagen, Hilden, Germany).

Additionally, midgut tissues obtained from both $O$. erraticus and O. moubata unfed females and fed females $48 \mathrm{hpf}$ were used to prepare protein extracts enriched in either soluble or membrane-associated proteins [23]. Briefly, batches of 25 midguts from each species and physiological condition were homogenised and sonicated in ice-cold phosphate-buffered saline (PBS) ( $\mathrm{pH} 7.4$ ) supplemented with proteinase inhibitors (Roche Diagnostics, Indianapolis, USA). The homogenates were centrifuged at $10^{4} \times g$ and the $10^{4} \mathrm{~g}$ supernatants, free of particulate material, were re-centrifuged at $10^{5} \times g$ into new supernatants and pellets containing, respectively, the soluble and membrane proteins. Protein concentration in the midgut extracts was assessed with the BCA Protein Assay Reagent kit (Thermo Fisher Scientific, Rockford, USA). The extracts were stored at $-20^{\circ} \mathrm{C}$ until use.

Tick saliva was collected from $O$. erraticus and $O$. moubata females following the protocol described by [46], that involved salivation stimulation with $1 \%$ pilocarpine. Protein concentration in the saliva samples was measured using the Bradford assay (Bio-Rad, Hercules, USA) and the samples were stored at $-20^{\circ} \mathrm{C}$.

\section{Analysis and selection of chitinases, tetraspanins, acidic ribosomal protein $\mathrm{P0}$ and secreted proteins/peptides}

Transcriptomic data regarding chitinases, tetraspanins, acidic ribosomal protein $\mathrm{P} 0$ and secreted proteins/peptides of $O$. erraticus, namely the transcript sequences and the transcription levels expressed in fragments per kilobase of transcript per million mapped reads (FPKM), were acquired from the $O$. erraticus midgut transcriptome, which has recently been obtained by our team [24]. The immunogenicity of the encoded proteins was predicted with the VaxiJen 2.0 software (http://www.ddgpharmfac.net/vaxijen/vaxijen/vaxijen.html) using the 0.5 
antigenicity threshold established by default for parasites [47-49].

One or two members from each of the above-mentioned protein families were chosen as potential protective candidate antigens. For this selection, proteins with the highest expression level, the highest fold-change after feeding and the highest predicted antigenicity (Vaxijen score) were prioritised. Additionally, the presence of these proteins in the O. erraticus midgut proteome [23] was also scored. For every selected candidate protein, its orthologues in argasid and ixodid ticks were searched in the Uniprot and NCBInr databases by BLASTp. The Clustal Omega alignment tool (https://www.ebi.ac.uk/ Tools/msa/clustalo/) was used to align multiple orthologous amino acid sequences and identify conserved protein regions. Phylogenetic analyses of the aligned proteins were performed using the MEGA v.6 package [50]. Phylogenetic trees were built using the neighbour-joining method, gaps were treated as pairwise deletions, amino acid distances were calculated using the Poisson model, and branch supports were assessed by bootstrap analysis (10,000 bootstraps).

Topographical predictions of the amino acid sequence of every selected candidate, including their transmembrane and extracellular exposed regions, were analysed with the TMHMM and SACS TMHMM software (http:// www.sacs.ucsf.edu/cgi-bin/tmhmm.py) [51, 52]. Additionally, the presence/absence of signal peptides, nonclassical secretion signals and GPI anchors was checked using the SignalP-5.0 server (http://www.cbs.dtu.dk/servi ces/SignalP/), the SecretomeP 2.0 server (http://www. cbs.dtu.dk/services/SecretomeP/) [53], and the GPISOM server (http://gpi.unibe.ch/) [54], respectively.

Prediction of secondary structures and three-dimensional (3D) modelling of the candidate proteins were done at the Phyre ${ }^{2}$ server [55]. The resulting 3D models were visualised using the Pymol package [56].

The presence of continuous linear B-cell epitopes on the selected candidate proteins was assessed using the following prediction tools: ABCpred (http://www.imtec h.res.in/raghava/abcpred/index.html) [57], BCEpred (http://www.imtech.res.in/raghava/bcepred/) [58] and BepiPred-2.0 (http://www.cbs.dtu.dk/services/BepiP $\mathrm{red} /$ ) [59]. The overlapping amino acid sequences in B-cell epitopes predicted by at least two of these tools were defined as the consensus predicted epitopes.

\section{Cloning of the candidates and production as recombinant proteins}

The cDNA sequences encoding the full-length candidate OeRPPO, a truncated version (without signal peptide) of candidate OePK4, and the extracellular exposed regions of candidates OeCHI, OeTSP1 and OeTSP2 were cloned and expressed as recombinant proteins.

With this aim, the corresponding cDNA coding sequences were amplified by RT-PCR from total RNA from the midgut. Table 1 shows the PCR conditions for these amplifications and the specific primer pairs designed ad-hoc, which included suitable restriction sites to assist with subcloning into the $\mathrm{pQE}-30$ (Qiagen) or pGEX-4T1 (GE Healthcare, Chicago, USA) expression vectors. The PCR products were first cloned in the pSC-A vector (Stratagene, LaJolla, USA) to verify their sequences. After that, they were digested and subcloned into the corresponding expression vector following previously described standard procedures $[60,61]$.

Recombinant $\mathrm{pQE}-30$ plasmids containing the cDNA fragments coding for OePK4, OeRPP0 and OeTSP2 were transformed into E. coli M15 cells (Qiagen) and protein expression was induced with $1 \mathrm{mM}$ IPTG following standard procedures. All of these proteins were expressed in $100 \%$ insoluble form. Thus, they were solubilised with $8 \mathrm{M}$ urea, purified by nickel affinity chromatography in denaturing conditions, and dialysed against PBS (pH 7.4), for $24 \mathrm{~h}$ at $4{ }^{\circ} \mathrm{C}$ according to the procedure described by [61].

Recombinant pGEX-4T-1 plasmids containing the cDNA fragments coding for OeCHI and OeTSP1 were transformed into Escherichia coli BL21 cells and protein expression was induced with $0.1 \mathrm{mM}$ IPTG. The CHIGST and TSP1-GST fusion proteins were expressed in the $100 \%$ insoluble form. Therefore, they were solubilised with $8 \mathrm{M}$ urea from the cellular lysate pellet and purified by electroelution from SDS-PAGE gels following the procedure described by [62]. The purified recombinant proteins were checked by SDS-PAGE and their identity was confirmed by in-gel enzymatic digestion followed by liquid chromatography and tandem mass spectrometry (LC-MS/MS) in a similar way as described in [23]. The concentration of the purified proteins was assessed by band densitometry in Coomassie blue-stained polyacrylamide gels and interpolation into a bovine serum albumin (BSA) standard curve. Purified proteins were stored at $-20^{\circ} \mathrm{C}$.

\section{Vaccine trial 1}

The aim of this trial was to assess the capacity of the candidate recombinant antigens to induce protective immune responses in rabbits against infestations by $O$. erraticus and O. moubata ticks.

Except candidates TSP1-GST and TSP2, which were formulated together, each candidate antigen was individually formulated in Montanide ISA 50 V2 (Seppic, La Garenne Colombe Cedex, France) and administered to a group of three New Zealand white rabbits. Additionally, 
Table 1 Primers and PCR conditions used for amplification of the cDNA fragments encoding the target protein regions

\begin{tabular}{|c|c|c|c|c|c|c|}
\hline Target protein & Target region (aa) & Primer name & Sequence $\left(5^{\prime}-3\right)$ & Product size (bp) & Restriction enzymes & Expression vector \\
\hline \multirow[t]{2}{*}{$\mathrm{OeCHI}$} & \multirow[t]{2}{*}{$\begin{array}{l}\text { Extracellular domain } \\
\quad(23-467)\end{array}$} & Oe6961_BamH1_F & $\frac{\text { GGATCCCAGGACGGC }}{\text { GCCGCA }}$ & \multirow[t]{2}{*}{1350} & \multirow[t]{2}{*}{ BamH1; Sma1 } & \multirow[t]{2}{*}{ pGEX-4T-1 } \\
\hline & & Oe6961_Sma1_ & $\begin{array}{l}\text { CCCGGGCTAGCACATGCC } \\
\text { ATTGATGCATTTAT }\end{array}$ & & & \\
\hline \multirow[t]{2}{*}{ OeTSP1* } & \multirow[t]{2}{*}{$\begin{array}{l}\text { Long extracellular } \\
\text { domain (107-195) }\end{array}$} & Oe1520_BamH1_F & $\frac{\text { GGATCCAAGGTGGCT }}{\text { GACGGAGACA }}$ & \multirow[t]{2}{*}{282} & \multirow[t]{2}{*}{ BamH1; Sma1 } & \multirow[t]{2}{*}{ pGEX-4T-1 } \\
\hline & & Oe1520_Sma1_R & $\frac{\text { CCCGGGCTAGACAGC }}{\text { ACCCGTTCTCTCC }}$ & & & \\
\hline \multirow[t]{2}{*}{ OeTSP2 } & \multirow[t]{2}{*}{$\begin{array}{l}\text { Long extracellular } \\
\text { domain (124-213) }\end{array}$} & Oe8446_BamH1_F & $\begin{array}{l}\text { GGATCCGCCTACGTCTCA } \\
\text { ACGTCCA }\end{array}$ & \multirow[t]{2}{*}{285} & \multirow[t]{2}{*}{ BamH1; Smal } & \multirow[t]{2}{*}{ pQE-30 } \\
\hline & & Oe8446_Sma1_R & $\begin{array}{l}\text { CCCGGGCTATGAGAGTCC } \\
\text { ATTGGATCGCA }\end{array}$ & & & \\
\hline \multirow[t]{2}{*}{ OePK4 } & \multirow[t]{2}{*}{$\begin{array}{l}\text { Truncated, without signal } \\
\text { P (22-109) }\end{array}$} & tOe9280_BamH1_F & $\frac{\text { GGATCC AGGCCTACC }}{\text { GAATCCGAGT }}$ & \multirow[t]{2}{*}{279} & \multirow[t]{2}{*}{ BamH1; Smal } & \multirow[t]{2}{*}{ pQE-30 } \\
\hline & & Oe9280_Sma1_R & $\frac{\text { CCCGGGTTACTGGAG }}{\text { AGGGATCTCTAC }}$ & & & \\
\hline \multirow[t]{2}{*}{ OeRPPO } & \multirow[t]{2}{*}{ Whole protein (1-319) } & OeRPP0_BamH1_F & $\begin{array}{l}\text { GGATCCATGGTCAGGGAG } \\
\text { GATAAGACA }\end{array}$ & \multirow[t]{2}{*}{972} & \multirow[t]{2}{*}{ BamH1; Hind3 } & \multirow[t]{2}{*}{ pQE-30 } \\
\hline & & OeRPP0_Hind3_R & $\frac{\text { AAGCTTCTAATCAAAAAG }}{\text { TCCGAAGCCCA }}$ & & & \\
\hline
\end{tabular}

Notes: Primers include suitable restriction sites (underlined) to assist in the sub-cloning into the corresponding expression vector (last column)

PCR conditions were the same for all targets $\left(94^{\circ} \mathrm{C} 3 \mathrm{~min} ; 10 \times\left(94^{\circ} \mathrm{C}\right.\right.$ for $15 \mathrm{~s} 59.5^{\circ} \mathrm{C}$ for $30 \mathrm{~s}, 72{ }^{\circ} \mathrm{C}$ for $\left.40 \mathrm{~s}\right)+25 \times\left(94{ }^{\circ} \mathrm{C}\right.$ for $15 \mathrm{~s}, 66^{\circ} \mathrm{C}$ for $30 \mathrm{~s} ; 72^{\circ} \mathrm{C}$ for $\left.40 \mathrm{~s}\right) ; 72{ }^{\circ} \mathrm{C}$ for $7 \mathrm{~min}$ ), except for TSP1, which required $60.5^{\circ} \mathrm{C}$ in the first 10 amplification rounds

two groups of rabbits were included as controls: one group was treated with recombinant GST from Schistosoma japonicum (SjGST; Sigma-Aldrich, Saint Louis, USA) formulated in Montanide ISA $50 \mathrm{~V} 2$ and the other group was treated with the adjuvant alone. Each animal received three doses of $100 \mu \mathrm{g}$ of the corresponding recombinant antigen administered subcutaneously at 15-day intervals.

Rabbits were bled immediately before administration of the first antigen dose (pre-immune sera), 14 days post-immunisation and immediately before tick infestation (14 dpi sera), and 14 days after the infestation (28 dpi sera). Blood samples were allowed to clot and sera were removed and stored at $-80^{\circ} \mathrm{C}$.

In the immune sera, their antibody titre to the homologous antigen and their reactivity to the other antigens were checked in ELISA following standard procedures [63]. Briefly, the ELISA plates were coated with $100 \mathrm{ng} /$ well of recombinant antigen in $100 \mu \mathrm{l} /$ well of carbonate buffer (pH 9.6), at $4{ }^{\circ} \mathrm{C}$ overnight, and post-coated with $1 \%$ BSA in PBS for $1 \mathrm{~h}$ at $37^{\circ} \mathrm{C}$. The sera were diluted in TPBS (PBS supplemented with $0.05 \%$ Tween 20) in a two-fold dilution series starting at $1 / 100$, and each dilution was incubated in duplicate wells at $37^{\circ} \mathrm{C}$ for $1 \mathrm{~h}$. Peroxidase-conjugated anti-rabbit IgG (Sigma-Aldrich) was diluted $1 / 10,000$ in TPBS and incubated at $37{ }^{\circ} \mathrm{C}$ for $1 \mathrm{~h}$. Ortho-phenylene-diamine (OPD) (Sigma-Aldrich) was used as a chromogen substrate and the reactions were stopped with $3 \mathrm{~N}$ sulphuric acid. The highest dilution of the immune serum giving more than twice the reactivity of the corresponding pre-immune serum at the same dilution was taken as the serum titre.

Once titrated, the immune sera were reacted in ELISA and western blot to tick saliva and the four midgut protein extracts (soluble and membrane proteins from fed and unfed females) from $O$. erraticus and $O$. moubata [22]. The ELISA plates were coated with $1 \mu \mathrm{g}$ of saliva or midgut extract per well, the sera were diluted $1 / 300$ in TPBS and the PO-anti-rabbit IgG was diluted 1/10,000.

Fourteen days after the last antigen dose, batches of 15 females, 30 males and 50 nymphs- 3 of O. erraticus, and 15 females, 30 males and 50 nymphs- 3 of O. moubata were allowed to feed on every rabbit for a maximum of $2 \mathrm{~h}$. After that time, any tick still remaining on the animal was removed. To estimate the degree of protection, the following parameters were measured: the amount of blood ingested by every developmental stage analysed; the female oviposition and fertility rates (respectively, the number of eggs laid per female and newly hatched larvae/ nymphs-1 per female); the moulting rate of nymphs-3; and the mortality rates of all the developmental stages tested.

The obtained data were subjected to statistical analysis using SPSS Statistics v25 software (IBM, Armonk, USA). For every parameter, the obtained values in the parasites that fed on each group of animals were summarised as 
the mean \pm standard deviation. The overall differences among groups were compared by a one way-ANOVA. When global differences were detected in this analysis, a post-hoc test (bilateral Dunnett's T-test) was applied to compare each vaccinated group to the merged control groups (adjuvant and $\mathrm{SjGST}$ ) treated as a single control group. All statistical analyses were considered significant at the $p<0.05$ level.

For each antigen formulation, the vaccine efficacy (E) was calculated according to the formula established by [64], and later updated by [65, 66]; this is based on comparing the reduction in the studied developmental processes between ticks fed on vaccinated animals and ticks fed on controls. Here, vaccine efficacy was calculated as $\mathrm{E}=100 \times[1-(\mathrm{S} \times \mathrm{F} \times \mathrm{N} \times \mathrm{M})]$, where $\mathrm{S}$ and $\mathrm{F}$ are the reduction in the survival and fertility of female ticks, respectively, and $\mathrm{N}$ and $\mathrm{M}$ represent the reduction in survival and moulting of nymphs-3, respectively.

\section{Vaccine trial 2}

The goal of this trial was to assess the combined vaccine efficacy of the more protective candidate antigens tested in the trial 1 (OeTSP1, OeTSP2, OeRPP0, OePK4) and the synthetic immunogenic peptide OeSEL, derived from the $O$. erraticus selenoprotein $\mathrm{T}$, which was the more protective candidate in our preceding study [25].

For this, multi-antigenic formulation doses containing $100 \mu \mathrm{g}$ of each candidate antigen in $1 \mathrm{ml}$ of PBS were emulsified in an equal volume of Montanide ISA $50 \mathrm{~V} 2$ and administered to a group of three rabbits, following the same procedures as previously described for trial 1 for the assessment of cocktail efficacy. One additional group of rabbits was treated with Montanide ISA 50 V2 alone and used as the control.

\section{Results}

\section{Chitinases: selected candidate $\mathrm{OeCHI}$}

Thirty-two transcripts annotated as chitinase and/or glycoside hydrolase-18 (GH-18 family) were recovered from the $O$. erraticus midgut transcriptome. Eleven of these transcripts were upregulated upon feeding (foldchange $>2$ ) and five of them were also predicted to be antigenic (Vaxijen score $>0.5$ ). Among the latter, transcript ci|000016961 showed by far the highest expression levels in the tick midgut, both before and after feeding (Additional file 1: Table S1). This transcript encodes a 492 amino acid long protein, which was also detected in the O. erraticus midgut proteome [23]. This transcript/ protein was selected as a candidate antigen and termed OeCHI.

Uniprot and NCBInr databases searching for tick orthologues of OeCHI retrieved 12 top matches, comprising 3 argasid and 9 ixodid chitinases belonging to the GH-18 family. All of them showed E-values $<10^{-70}$ and amino acid sequence identities among 35\% and $41 \%$ with sequence coverage between $64 \%$ and $78 \%$. The amino acid sequence alignment of these 12 chitinases and $\mathrm{OeCHI}$ showed some conservation along the GH18 catalytic domain (amino acids 35-398 in OeCHI), which typically comprises four signature amino acid sequence motifs in arthropod chitinases, including the active site "FDG(L/F)DLDWE(Y/F)P" [27]. Outside the CH18 domain, the OeCHI showed no sequence conservation (Additional file 2: Figure S1a).

The phylogenetic analysis of these tick $\mathrm{GH} 18$ chitinases clustered them into two main clusters, A and B, supported by $80 \%$ and $96 \%$ bootstrap values, respectively, whereas the $\mathrm{OeCHI}$ remained outside these clusters (Fig. 1a).

Topology prediction for OeCHI showed a single spanning transmembrane protein with a 22 amino acid long signal peptide, a large extracellular domain (aa 23-467) that contains the GH18 catalytic domain, a carboxy-terminal transmembrane domain (aa 468-490) and a very short cytoplasmic tail (aa 491-492) (Additional file 2: Figure S1b).

Linear B-cell epitope predictions for $\mathrm{OeCHI}$ are shown in Fig. 1b. Each immunoinformatics tool predicted a set of distinct but overlapping linear B-cell epitopes. Up to 7 epitopes were predicted by two or three algorithms, and their overlapping sequences were considered as the final predicted linear B-cell epitopes. Six of these epitopes were distributed throughout the GH-18 catalytic domain, actually covering the active site (Fig. 1b).

The three-dimensional (3D) modelling of OeCHI modelled up to 352 residues (72\%) of its amino acid sequence with $100 \%$ confidence by the single highest scoring 3D template. The resulting 3D model showed the typical 3D structure for the catalytic region of GH-18 chitinases [67], i.e. the $(\alpha / \beta) 8$-TIM-barrel, which comprises 8 parallel $\beta$ sheets forming a barrel in turn surrounded by $8 \alpha$ helices that form a ring towards the outside (Additional file 2: Figure S1c). The six linear B-cell epitopes predicted for the GH-18 domain were located on the surface of OeCHI 3D model, where they could be easily accessed by host antibodies (Additional file 2: Figure S1c). The seventh predicted epitope could not be localised on the 3D model because it was close to the carboxy-end, outside the modelled region. For this protein, the whole extracellular region of OeCHI (aa 23-467) was cloned and produced as a recombinant candidate antigen (see below).

\section{Tetraspanins: selected candidates OeTSP1 and OeTSP2}

Seventeen transcripts annotated as Tetraspanin (TM4SF) family members were recovered from the $O$. erraticus midgut transcriptome, but only seven were full length 


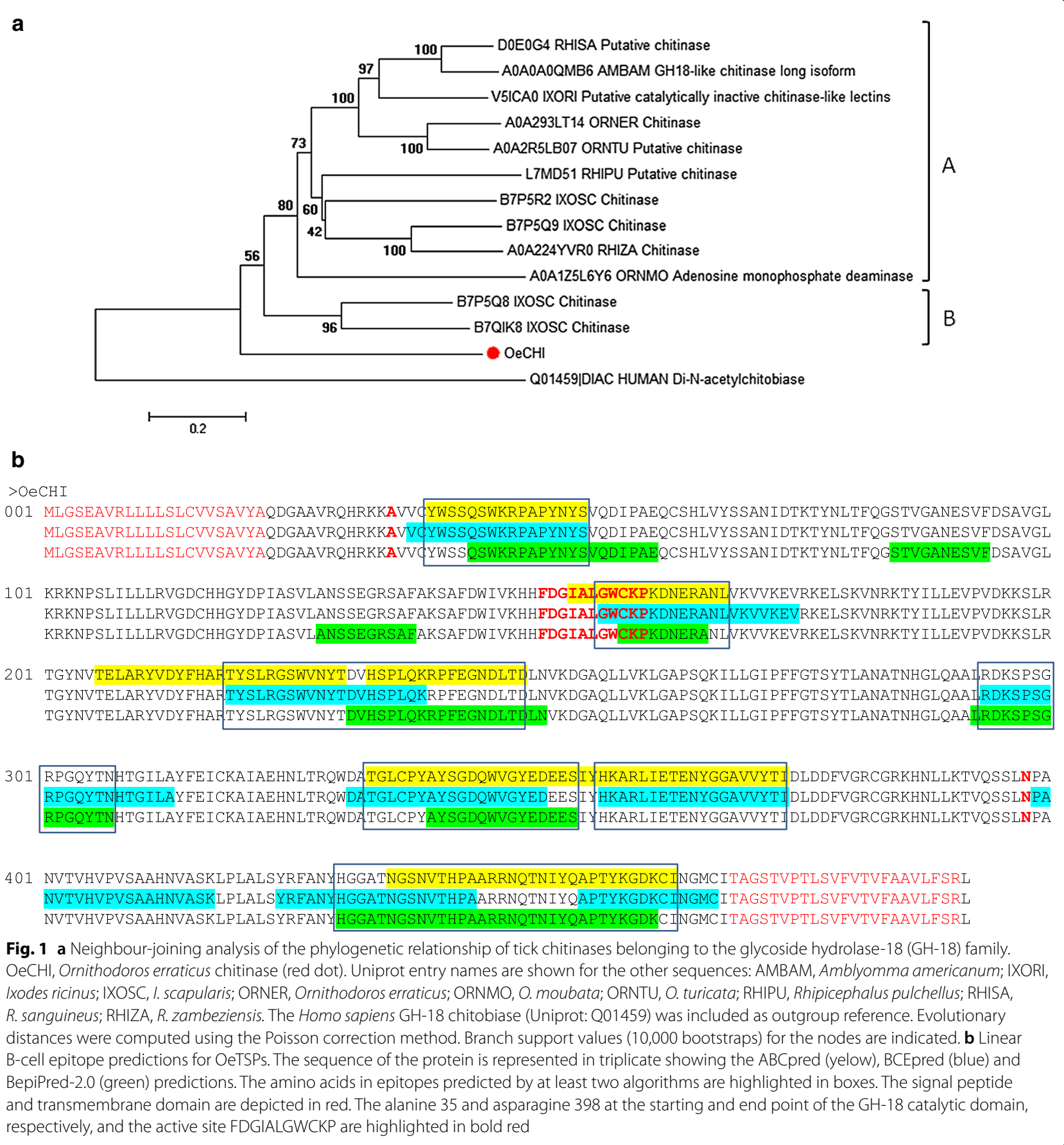

tetraspanins (200-350 amino acid residues) displaying the characteristic four transmembrane domains $[32,34]$. All of them showed fold-changes after feeding between -2.1 and 2.4, indicating that they were slightly or not differentially expressed upon feeding. Among them, four were predicted to be antigenic (Vaxijen scores $>0.5$ ) and the two most antigenic also showed the highest expression levels in tick midgut both before and after feeding: namely, transcripts ci|000077740 (Uniprot: A0A293MYE4) and ci|000018446 (Uniprot: A0A293M0B7) (Additional file 1: Table S1). These transcripts encoded two proteins of 226 and 246 amino acid long, respectively, which were actually detected in the $O$. erraticus midgut proteome [23]. Hence, we selected both of them as candidate antigens and termed them OeTSP1 and OeTSP2, respectively. 
Searching Uniprot and NCBInr databases for tick orthologues of OeTSP1 retrieved 12 tick sequences; eight of them were full-length, highly conserved argasid and ixodid tetraspanins, with E-values $<10^{-120}$ and more than $85 \%$ sequence identity (Additional file 3: Figure S2a). BLASTp searching for tick orthologues of OeTSP2 retrieved 10 tick sequences including full-length and fragment tetraspanins, which displayed E-values $<10^{-33}$ and a sequence identity between $30 \%$ and $58 \%$ (Additional file 3: Figure S2b). OeTSP1 and OeTSP2 had 27.3\% sequence identity with each other (Additional file 3: Figure S2c).

The phylogenetic analysis of these TSPs showed a very close relationship between OeTSP1 and its orthologues, grouping them into one tight cluster supported by a $99 \%$ bootstrap value. The same analysis placed OeTSP2 and its orthologues into two different clusters, supported by $87 \%$ and $98 \%$ bootstrap values, which were poorly related to the OeTSP1 cluster (Fig. 2a).

Topological prediction for OeTSP1 and OeTSP2 confirmed that both are typical four-transmembrane tetraspanins with the characteristic short (13-18 amino acids) and long (88-89 amino acids) extracellular loops (Additional file 3: Figure S2d).

Linear B-cell epitope predictions are shown in Fig. 2b. For OeTSP1, the three algorithms predicted two large epitopes of 29 and 36 amino acids each that cover most of the sequence of the long extracellular loop, while the rest of the protein had no significant predictions. For OeTSP2, the three algorithms predicted four epitopes of 8, 24, 32 and 11 amino acids, covering almost the entire length of its long extracellular loop. Additionally, they also predicted two more epitopes on the cytoplasmic Nand C-termini, respectively.

3D modelling of OeTSP1 and OeTSP2 modelled up to 220 and 219 residues (97\% and 89\%), respectively, of their amino acid sequences with $100 \%$ confidence by the single highest scoring 3D template. The resulting 3D models displayed the typical 3D structure for monomeric Tetraspanin (TM4SF) family members, and showed the predicted epitopes on the surface of the long extracellular loops of both molecules, where they could be easily accessed by host antibodies (Additional file 3: Figure S2e). Hence, the two long extracellular loops were cloned and produced as recombinant candidate antigens (see below).

\section{Acidic ribosomal protein PO (RPPO): candidate OeRPPO}

Two transcripts annotated as the $60 \mathrm{~S}$ acidic ribosomal protein $\mathrm{P} 0$ were recovered from the $\mathrm{O}$. erraticus midgut transcriptome (Additional file 1: Table S1), but only one of them (ci|000113905) was upregulated upon feeding (fold-change, 2.78) and encoded a full-length ribosomal protein P0, namely, a 319 amino acid long protein without signal peptide, non-classical secretion signals, transmembrane domains or GPI anchors. This protein, termed OeRPP0, was also detected in the O. erraticus midgut proteome [23] and was selected as a vaccine candidate despite its predicted antigenicity being below the threshold (VaxiJen score 0.444).

Uniprot and NCBInr databases searching for tick orthologues of OeRPP0 retrieved 20 highly conserved tick sequences, all of them with an E-value $=0$ and more than $90 \%$ sequence identity. Multiple alignment of these proteins confirmed their high conservation degree, including the 8 amino acid residues that form the $28 \mathrm{~S}$ rRNA interface and the 20 residues that constitute the putative interface with the L7/L12 ribosomal proteins (Additional file 4: Figure S3a).

The phylogenetic analysis confirmed the close relationship among all of these RPPOs and grouped them into three main clusters, which respectively include the RPP0 from Metastriata (A), Prostriata (B) and Argasidae (C), supported by $70 \%, 99 \%$ and $99 \%$ bootstrap values (Fig. 3a).

Each lineal B-cell epitope prediction algorithm identified a set of different but overlapping linear B-cell epitopes throughout the whole protein sequence. Up to 7 epitopes were predicted by two or the three algorithms, and thus considered consensus predicted linear B-cell epitopes. The longest epitope spanned 26 amino acid residues close to the carboxy-end, inside a highly unstructured region of the protein (Fig. 3b).

The 3D modelling of OeRPP0 modelled up to 269 residues (84\%) of its amino acid sequence with $100 \%$ confidence by the single highest scoring 3D template. Additional file 4: Figure S3b presents the 3D model of OeRPP0, showing the highly conserved secondary structure of RPP0s and the predicted linear B-cell epitopes localised on the protein surface, where they cover the whole putative interface with the 23S rRNA and most part of the interface with the L7/L12 ribosomal proteins. The longest predicted epitope could not be included in the 3D model because it mapped outside the modelled region. For this protein, the whole amino acid sequence was cloned and expressed as recombinant candidate antigen.

Secreted peptides and proteins: selected candidate OePK4 Forty-six transcripts annotated as "secreted peptide" or "secreted protein" were mined from the O. erraticus midgut transcriptome, very few of them having functional annotation (Additional file 1: Table S1). Twentyone were significantly upregulated (fold-change $>2$ ) after feeding and 11 of them encoded antigenic proteins (Vaxijen score $>0.5$ ). Among the predicted antigens, 
a
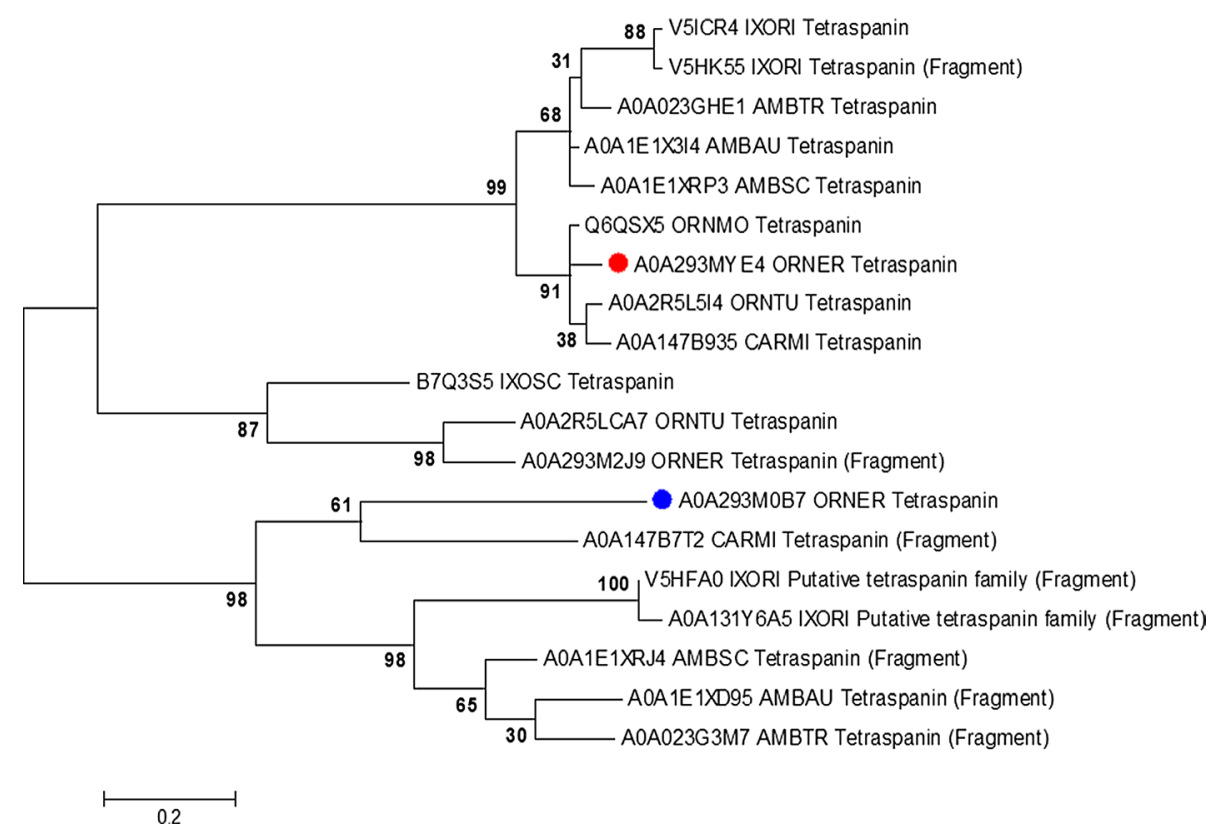

b

$>$ OeTSP 1

001 MDGGIACVKYVLIACNLLVWILGLGVLSIGIWIRSDPDFWIYQDNLPLSNYYDACYVIMAVGVLLLVLGFMGCCAAAIDSPCMLLTYFIAMLALLLMECA MDGGIACVKYVLIACNLLVWILGLGVLS IGIWIRSDPDFWIYQDNLPLSNYYDACYVIMAVGVLLLVLGFMGCCAAAIDSPCMLLTYFIAMLALLLMECA MDGGIACVKYVLIACNLLVWILGLGVLS IGIWIRSDPDFWIYQDNLPLSNYYDACYVIMAVGVLLLVLGFMGCCAAAIDSPCMLLTYFIAMLALLLMECA i i i i i i i i i iMMMMMMMMMMMMMMMMMMMMMMo000000000000000000MMMMMMMMMMMMMMMMMMMM i i i i iMMMMMMMMMMMMMMMMMMM

101 VAGLVWKVA DGDTLQRYLATTITEKIDE INENPKTRRFMDLMQVHLECCGAISKHDYEVRAMTI PQSCSSSRTNNIFIYGCSENLRVLLERTGAVVGGMG VAGLVWKVADGDTLQRYLATTITEKIDE INENPKTRRF MDLMQVHLECCGAISKHDYEVRAMTI PQSCSSSRTNNIF IYGCSENLRVLLERTGAVVGGMG VAGLVWKVADGDTLQRYLATTITEKIDEINENPKTRRFMDLMQVHLECCGAISKHDYEVRAMTI PQSCSSSRTNNIFIYGCSENLRVLLERTGAVVGGMG MMMMMM0000000000000000000000000000000000000000000000000000000000000000000000000000000000000000000MMMMM

201 LALGFVQIIVMIISLCLFCTLRQDGK

LALGFVQI IVMI ISLCLFCTLRQDGK

LALGFVQI IVMI ISLCLFCTLRQDGK

MMMMMMMMMMMMMMMMMMiiiiiii

$>0$ TSP2

001 MADEEAQRTTGGGSGYKRPPKSQSKCIKFTLIATNSVIWLLGLTVFILSVVLICA RHTSTELPAVQSLRDAAITGIVLGLILFCTGFLGCCGAAKENVCM MADEEAQRTTGGGSGYKRPPKSQSKCIKFTLIATNSVIWLLGLTVFI LSVVLICARHTSTELDAVQSLRDAAITGIVLGLILFCTGFLGCCGAAKENVCM MADEEAQRTTGGGSGYKRPPKSQSKCIKFTLIATNSVIWLLGLTVFILSVVLICARHTSTELDAVQSLRDAAITGIVLGLILFCTGFLGCCGAAKENVCM iiiiiiiiiiiiiiiiiiiiiiijiiiii MMMMMMMMMMMMMMMMMMMMMo0000000000000MMMMMMMMMMMMMMMMMMMMMMMiiiii

101 LATYFIILAVALI IEIAVMALALAYVSTSKLDDIVTVAF QMIKGGSREDTELLYSTQQNMROCGATGIDDYAGHDMPVPPSCFDSSEDNRSYIYADGCV LATYF I I LAVALI IEIAVMALALAYVSTSKLDDIVTVAF PQMIKGGSREDTELLYSTQQNMRCCGATGIDDYAGHDMPVP PSCFDSSEDNRSYIYAD GCV LATYFI I LAVALI IE IAVMALALAYVSTSKLDDIVTVAF PQMIKGGSREDTELLYSTQQNMRCCGATGIDDYAGHDMPVPPSCFDSSEDNRSYIYAD GCV MMMMMMMMMMMMMMMMMMMMMMMO00000000000000000000000000000000000000000000000000000000000000000000000000000

201 TALKHYLRSNGLSIGLIAFFTFFAQVCSMAGAAVIIKRKPRGKLTP TALKHYLRSNGLSIGLIAFFTFFAQVCSMAGAAVIIKRKPRGKLTP TALKHYLRSNGLSIGLIAFFTFFAQVCSMAGAAVIIKRKPRGKLTP o00000000000 00MMMMMMMMMMMMMMMMMMMMMMi i i ii iiij

Fig. 2 a Neighbour-joining analysis of the phylogenetic relationship of A0A293MYE4_ORNER (OeTSP1, red dot) and A0A293M0B7_ORNER (OeTSP2, blue dot) with their tick orthologues. Uniprot entry names are shown: AMBAU, Amblyomma aureolatum; AMBSC, A. sculptum; AMBTR, A. triste; CARMI, Carios mimon; IXORI, Ixodes ricinus; IXOSC, I. scapularis; ORNER, Ornithodoros erraticus; ORNMO, O. moubata; ORNTU, O. turicata. Evolutionary distances were computed using the Poisson correction method. Branch support values (10,000 bootstraps) for the nodes are indicated. b Linear B-cell epitope predictions for OeTSPs. The sequences of the proteins are represented in triplicate showing the ABCpred (yellow), BCEpred (blue) and BepiPred-2.0 (green) predictions. Epitopes predicted by at least two algorithms were highlighted in boxes. The predicted topology is indicated below the protein sequences to show that most epitopes mapped on the long extracellular domain: o (outside), extracellular; M, transmembrane; i, intracellular 


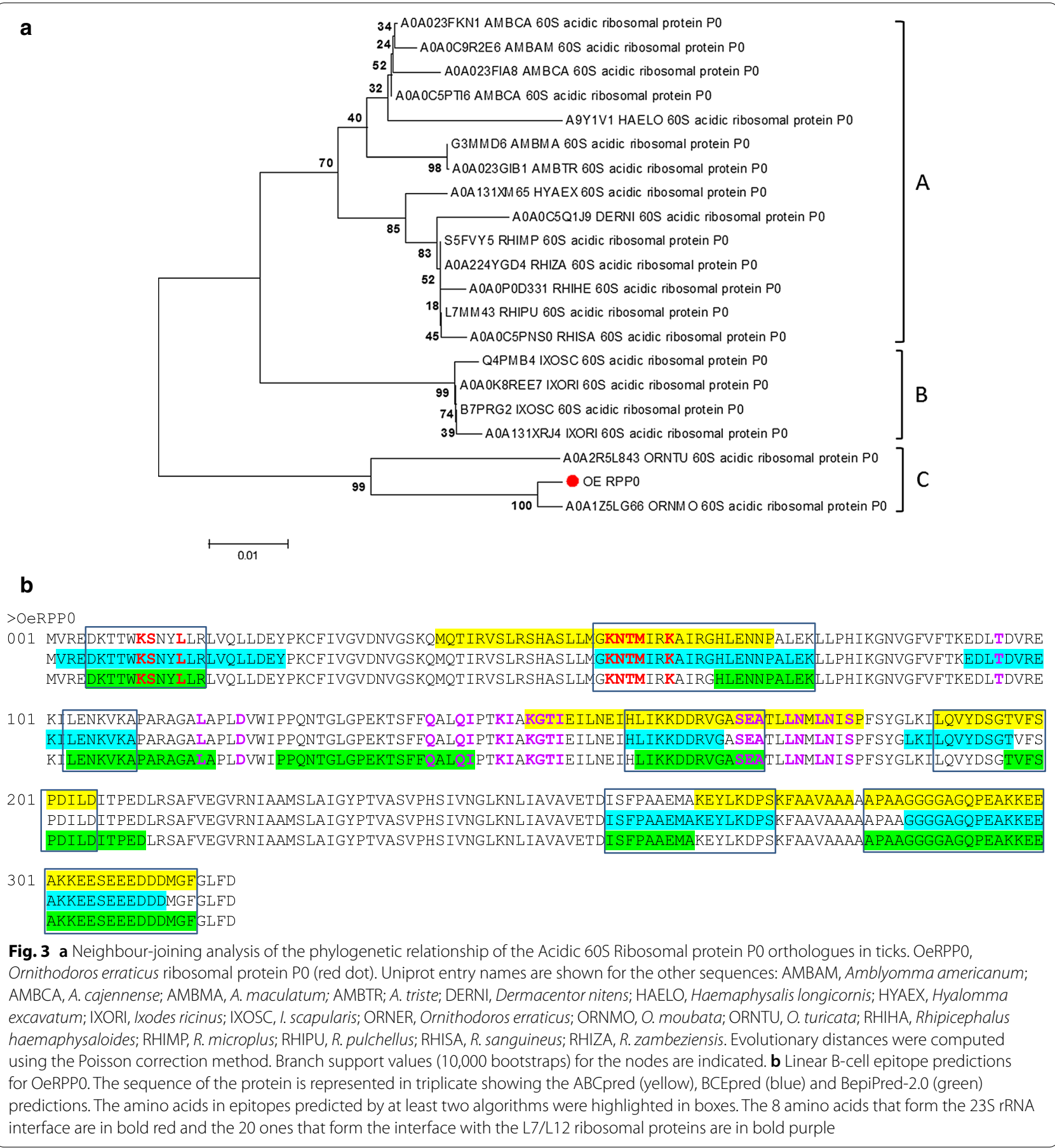

transcript ci|000079280 showed simultaneously the highest fold-change upon feeding (3954) and the highest Vaxijen score (1.0632). Accordingly, it was selected as candidate protective antigen. This transcript encodes a 109 amino acid long polypeptide without functional annotation (Uniprot: A0A293MVU8).
Searching Uniprot and NCBInr databases for tick orthologues of A0A293MVU8 provided numerous related sequences, from which the top 10 matches were selected; they all showed E-values $<10^{-7}$ and sequence identities between $32 \%$ and $58 \%$, and included 4 argasid and 6 ixodid sequences. Multiple alignment 
of A0A293MVU8 and these proteins showed that A0A293MVU8 has a poorly conserved amino-terminal region (residues 1-57) and a more conserved carboxyterminal region (residues 58-109), reaching in this region more than $50 \%$ sequence identity to the Q4PMD7, B7PVH8 and B7PUK6 proteins of Ixodes scapularis (Additional file 5: Figure S4).

Phylogenetic analysis of these proteins grouped them in two main clusters, supported by $97 \%$ and $98 \%$ bootstrap values, but placed A0A293MVU8 outside these clusters (Fig. 4a). None of these proteins were found in the Pfam, Prosite and InterPro databases of protein families, domains and functional sites (data not shown). However, all of them but B7P261 and Q4PMD7 belong to the Uniprot "Uniref_cluster: Cytochrome $c$ oxidase assembly protein", whose representative member is B7PVH8. Sequence identity between A0A293MVU8 and B7PVH 8 is $57.8 \%$, which might suggest a functional relation of A0A293MVU8 with the mitochondrial respiratory chain and redox-linked proton pumping. Despite this, since most of the A0A293MVU8 orthologues found were annotated as "secreted protein PK4", we termed A0A293MVU8 as OePK4.

Topology predictions for OePK4 predicted a secreted polypeptide with a signal peptide of 21 amino acids long, without transmembrane domains or GPI anchors. 3D modelling of OePK4 only modelled 16 amino acids (15\%) with $29.1 \%$ confidence, thus it was no longer considered (not shown).

Linear B-cell epitope prediction tools forecasted two epitopes for OePK4. The first epitope was located immediately downstream the signal peptide and the second in the carboxy-terminal half of the protein, where OePK4 shows the higher sequence identity to their orthologues in other tick species (Fig. 4b; Additional file 5: Figure S4). Thus, a truncated version of OePK4 without signal peptide was cloned and expressed as recombinant candidate antigen.

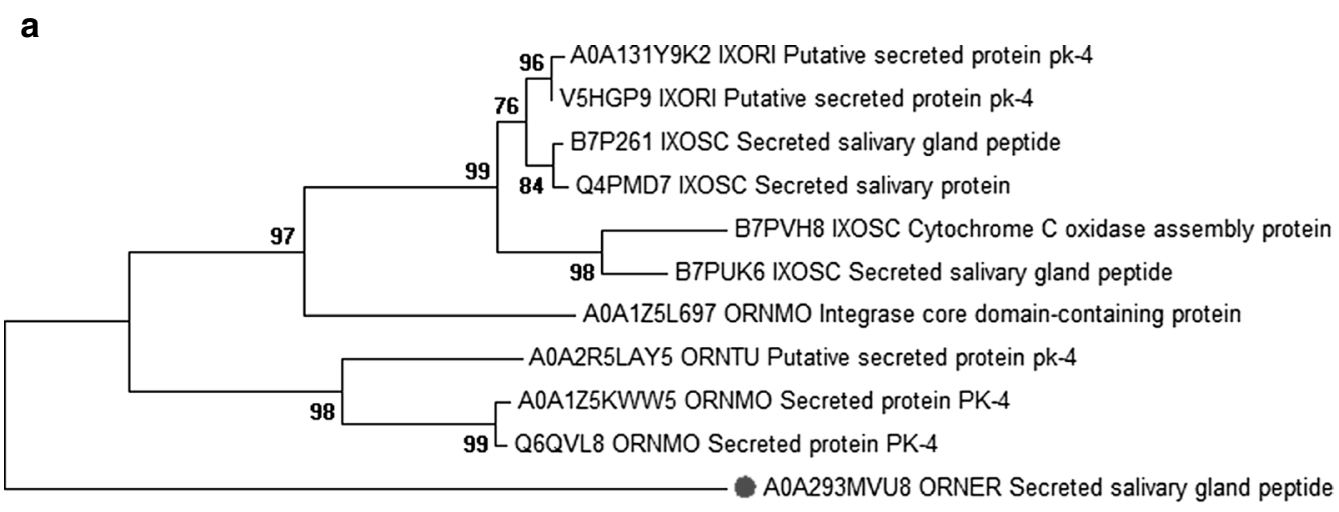

0.1

b

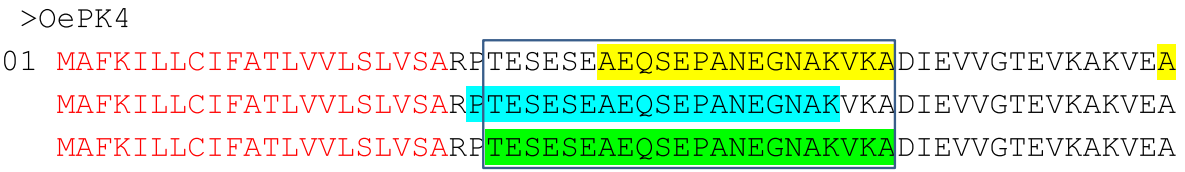

61 FLQGDLYRFENGGKVSGHVKGSQSFSSKDGGTWGSPQVEAGVEVEIPLQ FLQGDLYRFENGGKVSGHVKGSQSFSSKDGGTWGS PQVEAGVEVEIPLQ FLQGDLYRFENGGKVSGHVKGSQSFSSKDGGTWGSPQVEAGVEVEIPLQ

Fig. 4 a Neighbour-joining analysis of the phylogenetic relationship of A0A293MVU8_ORNER (OePK4, red dot) and orthologous proteins. Uniprot entry names are shown: IXORI, Ixodes ricinus; IXOSC, I. scapularis; ORNER, Ornithodoros erraticus; ORNMO, O. moubata; ORNTU, O. turicata. Evolutionary distances were computed using the Poisson correction method. Branch support values (10,000 bootstraps) for the nodes are indicated. $\mathbf{b}$ Linear B-cell epitope predictions for OePK4. The sequence of the protein is represented in triplicate showing the ABCpred (yellow), BCEpred (blue) and BepiPred-2.0 (green) predictions. The amino acids in epitopes predicted by at least two algorithms are highlighted in boxes. The signal peptide is depicted in red 


\section{Recombinant protein production}

The five candidates selected were all subcloned and expressed into the $\mathrm{pQE}-30$ vector. However, the expression of CHI and TSP1 failed and they were subcloned and expressed on the pGEX-4T1 vector.

Finally, the CHI-GST and TSP1-GST fusion proteins, the full-length recombinant RPP0, the truncated version (without signal peptide) of PK4 and the long extracellular loop of TSP2 were all successfully expressed and purified (Additional file 6: Figure S5). All of them migrated in SDS-PAGE gels as single bands of the predicted molecular weight (MW), except PK4 and TSP2, which showed experimental MWs (13.5 and $16.5 \mathrm{kDa}$, respectively) somewhat larger than their predicted MWs (11.5 and $11.1 \mathrm{kDa}$, respectively). Hence, the identity of these recombinants was confirmed by LC-MS/MS mass spectrometry analysis of the corresponding gel band (not shown).

Vaccine trial 1: humoral immune response to recombinant antigens and protective effects against tick infestations

The rabbits vaccinated with the five recombinant antigens developed strong antibody responses to the homologous antigen. The sera obtained $14 \mathrm{dpi}$, immediately before the infestation, showed antibody titres higher than 1/12,800 and optical densities (OD) higher than 2.0. Control rabbits immunised with SjGST reacted to this recombinant with antibody titres close to $1 / 6400$ and OD around 1.5 (Fig. 5a).

The sera obtained 28 dpi (14 days post-infestation) reacted with almost identical intensity to the recombinant antigens than the sera obtained 14 dpi did, suggesting lack of cross-reactivity between the recombinant antigens and the salivary proteins inoculated during feeding (Fig. 5a). Conversely, no sera obtained $14 \mathrm{dpi}$ before the infestation reacted to the saliva of $O$. erraticus or $O$. moubata (Fig. 5b).

The reactivity of the rabbit IgG antibody response to native forms of the OeCHI, OeTSP1, OeTSP2, OeRPP0 and OePK4 proteins in the midgut extracts from $O$. erraticus and $O$. moubata was analysed in ELISA (Additional file 7: Figure S6) and western blot (Fig. 6). According to the ELISA results, the immune sera showed low reactivity to the midgut extracts of $O$. erraticus, and even lower reactivity to the midgut extracts of O. moubata (Additional file 7: Figure S6). Part of this reactivity was due to the nonspecific recognition of host IgG, which was also recognised by
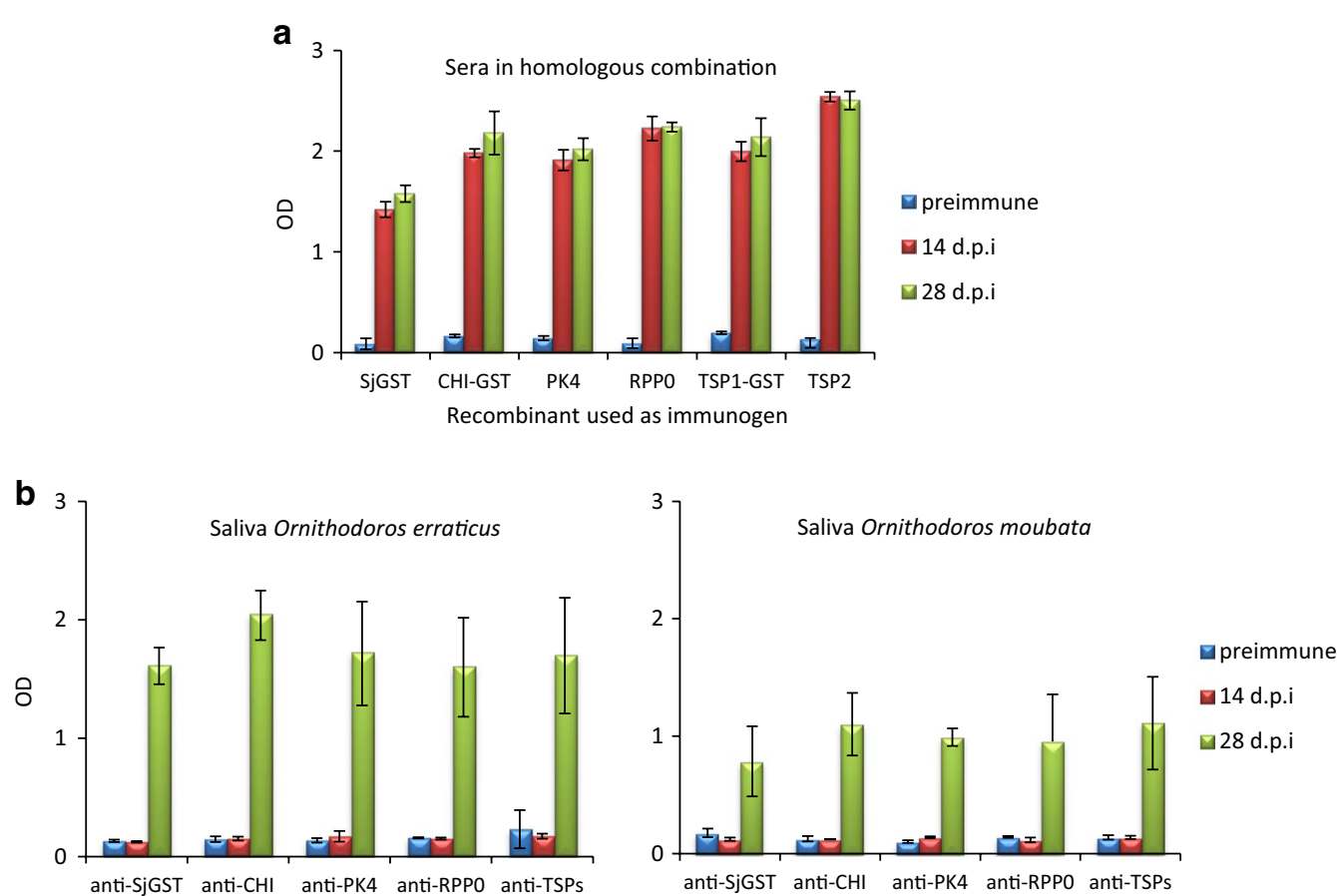

Fig. 5 ELISA. IgG antibody response in rabbits vaccinated with recombinant antigens SjGST (control), OeCHI, OePK4, OeRPPO and OeTSP1 + OeTSP2 (TSPS). a Reactivity of rabbit sera to the homologous recombinant antigen. $\mathbf{b}$ Reactivity of rabbit sera to the saliva of Ornithodoros erraticus and Ornithodoros moubata. Values are the average OD $\pm \mathrm{SD}$ at $492 \mathrm{~nm}$ from each rabbit group. Sera were taken before immunisation (preimmune), 14 days post-immunisation, immediately before the infestation with ticks ( $14 \mathrm{dpi}$ ) and 14 days post-infestation ( $28 \mathrm{dpi})$, and were used at $1 / 300$ dilution 

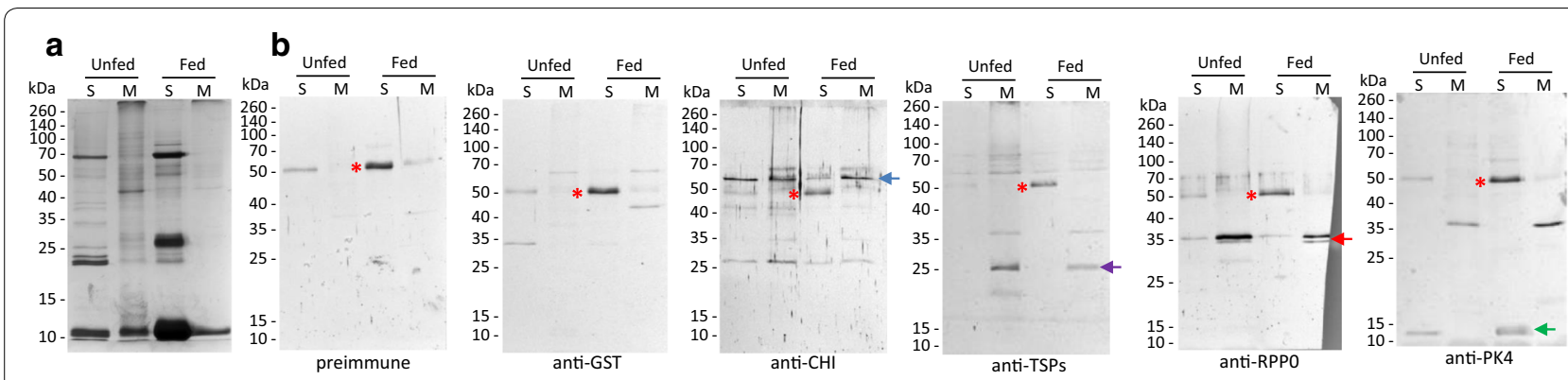

C

d
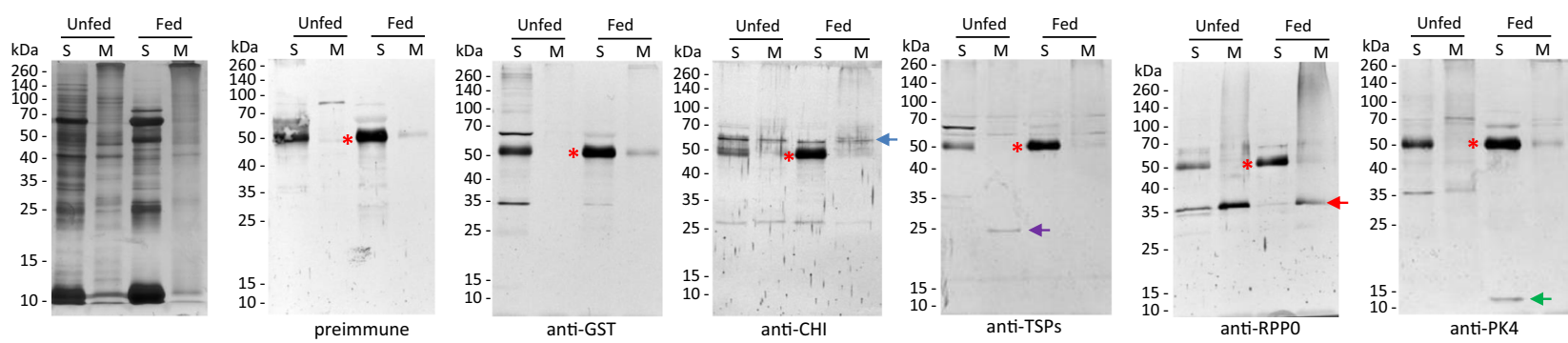

Fig. 6 a, c Coomassie Blue-stained 15\% SDS-PAGE gels showing the soluble (S) and membrane (M) proteins of the midgut from Ornithodoros erraticus (a) and O. moubata (c) female ticks taken before feeding (Unfed) and $48 \mathrm{~h}$ after engorgement (Fed). b, d Western blots: antigens revealed by the sera from the rabbits vaccinated with the recombinant antigens SjGST, OeCHI, OeTSP1 + OeTPS2 (TSPs), OeRPP0 and OePK4 on the protein extracts from 0 . erraticus (b) and O. moubata (d). Sera were taken before immunisation (preimmune) and 14 days post-immunisation, immediately before the infestation with ticks. Red asterisks: IgG heavy chain from the rabbit host ingested with blood. Arrows: native forms of OeCHI (55 kDa, blue), OeTSPs (25.9-26.4 kDa, purple) OeRPPO (34.7, red) and OePK4 (11.5, green) recognised by the immune sera

the preimmune sera and the sera anti-SjGST (Fig. 6). Overall, the immune sera showed higher reactivity to membrane proteins than to soluble proteins from both fed and unfed O. erraticus and O. moubata females, except for the anti-PK4 sera which showed the opposite behaviour, reacting more intensely to the soluble proteins (Fig. 6, Additional file 7: Figure S6).

On western blot, the anti-CHI serum pool reacted with a $55 \mathrm{kDa}$ band compatible with the native $\mathrm{OeCHI}$ protein in every protein extract analysed, namely, extracts of soluble and membrane proteins from fed and unfed O. erraticus (Fig. 6b) and O. moubata females (Fig. 6d). This indicates that OeCHI and its orthologue in O. moubata share cross-reacting epitopes and a similar localisation in the enterocyte, including in the plasma membrane and extracellular region or secreted.

The anti-TSPs serum pool reacted with a band of 26 $\mathrm{kDa}$, compatible with both the OeTSP1 and OeTSP2 native proteins, on the membrane proteins from fed and unfed $O$. erraticus and the membrane proteins from unfed O. moubata females. This is in agreement with the membrane predicted localisation of TSPs and also indicates cross-reactive epitopes between orthologous TSP proteins in these two Ornithodoros species.
The anti-RPP0 sera recognised a $34.7 \mathrm{kDa}$ intense band, compatible with the OeRPP0 native protein, on the extracts of membrane proteins of both Ornithodoros species and physiological stages (fed, unfed), and a similar but fainter band on the parallel extracts of soluble proteins (Fig. 6b, d). Thus, OeRPP0 and its native orthologue in O. moubata share cross-reacting epitopes and localisation in the enterocyte including cytosolic large ribosomal subunit, endoplasmic reticulum and extracellular region or secreted.

Finally, the anti-PK4 serum pool recognised one band of $11.5 \mathrm{kDa}$ compatible with the predicted native OePK 4 in the extracts of soluble proteins from fed and unfed $O$. erraticus (Fig. 6b) and soluble proteins from fed O. moubata (Fig. 6d), showing that OePK4 shares cross-reactive epitopes with orthologous proteins in O. moubata.

No significant differences were observed between the two control groups (adjuvant, SjGST) for any species and developmental stage in any of the parameters evaluated (Tables 2, 3). Accordingly, these two groups were combined and treated as a single control group.

On the O. erraticus ticks, the anti-CHI response caused a significant reduction on nymph moulting, while the anti-TSPs, anti-RPP0 and anti-PK4 responses induced 
Table 2 Effect of the vaccination with recombinant proteins CHI-GST, TSP1-GST + TSP2, RPP0, and PK4 administered in Montanide ISA $50 \mathrm{~V} 2$ on 0 . erraticus specimens fed on control and vaccinated rabbits

\begin{tabular}{|c|c|c|c|c|c|c|c|c|}
\hline Parameter & $\begin{array}{l}\text { Developmental } \\
\text { stage }\end{array}$ & $\begin{array}{l}\text { Control } \\
\text { Merged }\end{array}$ & $\begin{array}{l}\text { Control } \\
\text { Montanide }\end{array}$ & Control SjGST & $\begin{array}{l}\text { CHI-GST (\% } \\
\text { reduction) }\end{array}$ & $\begin{array}{l}\text { TSP1- } \\
\text { GST + TSP2 (\% } \\
\text { reduction) }\end{array}$ & $\begin{array}{l}\text { RPPO (\% } \\
\text { reduction) }\end{array}$ & $\begin{array}{l}\text { PK4 (\% } \\
\text { reduction) }\end{array}$ \\
\hline \multirow{3}{*}{$\begin{array}{l}\text { Ingested blood } \\
(\mathrm{mg})\end{array}$} & Males & $3.7 \pm 0.5$ & $3.7 \pm 0.1$ & $3.7 \pm 0.7$ & $3.6 \pm 0.1(2.7)$ & $3.8 \pm 0.2$ & $4.1 \pm 0.2$ & $3.4 \pm 0.3(8.1)$ \\
\hline & Females & $13.0 \pm 1.1$ & $13.0 \pm 3.1$ & $13.0 \pm 1.2$ & $13.3 \pm 0.6$ & $13.3 \pm 1.2$ & $12.8 \pm 2.0(1.5)$ & $12.5 \pm 0.2(3.8)$ \\
\hline & Nymphs-3 & $3.3 \pm 0.5$ & $3.2 \pm 0.4$ & $3.5 \pm 0.5$ & $3.0 \pm 0.5(9.1)$ & $3.9 \pm 0.4$ & $3.3 \pm 0.5$ & $3.1 \pm 0.7(6.1)$ \\
\hline \multirow[t]{3}{*}{ Mortality (\%) } & Males & $0.6 \pm 1.4$ & $0 \pm 0$ & $1.1 \pm 1.8$ & $0 \pm 0$ & $0 \pm 0$ & $0 \pm 0$ & $0 \pm 0$ \\
\hline & Females & $0 \pm 0$ & $0 \pm 0$ & $0 \pm 0$ & $0 \pm 0$ & $0 \pm 0$ & $2.1 \pm 3.0(2.1)$ & $2.1 \pm 3.0(2.1)$ \\
\hline & Nymphs-3 & $4.3 \pm 2.9$ & $6.0 \pm 2.0$ & $2.7 \pm 2.7$ & $1.3 \pm 1.0$ & $\begin{array}{l}10.0 \pm 12.9 \\
\quad(5.7)\end{array}$ & $1.4 \pm 1.0$ & $4.1 \pm 3.5$ \\
\hline Moulting (\%) & Nymphs-3 & $60.5 \pm 8.9$ & $61.1 \pm 9.8$ & $59.8 \pm 9.0$ & $\begin{array}{c}42.3 \pm 3.0 \\
(30.1)^{* * *}\end{array}$ & $\begin{array}{c}45.5 \pm 15.2 \\
(24.8)^{* *}\end{array}$ & $\begin{array}{c}38.1 \pm 10.7 \\
(37.1)^{* *}\end{array}$ & $\begin{array}{c}31.8 \pm 19.8 \\
(47.4)^{* *}\end{array}$ \\
\hline $\begin{array}{l}\text { Oviposition } \\
\text { (no. eggs/ } \\
\text { female) }\end{array}$ & Females & $66.4 \pm 6.0$ & $67.2 \pm 8.5$ & $65.6 \pm 3.5$ & $61.6 \pm 3.4(7.2)$ & $\begin{array}{c}50.0 \pm 4.5 \\
(24.7)^{* *}\end{array}$ & $\begin{array}{c}48.0 \pm 6.1 \\
(27.7)^{* *}\end{array}$ & $\begin{array}{c}54.2 \pm 6.0 \\
(18.4)^{* *}\end{array}$ \\
\hline $\begin{array}{l}\text { Fertility (no. } \\
\text { nymphs/ } \\
\text { female) }\end{array}$ & Females & $55.5 \pm 5.7$ & $56.8 \pm 6.3$ & $54.3 \pm 5.3$ & $53.7 \pm 1.6(3.2)$ & $\begin{array}{c}32.5 \pm 4.4 \\
(41.4)^{* *}\end{array}$ & $\begin{array}{c}36.6 \pm 2.7 \\
(34.1)^{* *}\end{array}$ & $\begin{array}{c}41.5 \pm 6.7 \\
(25.2)^{* *}\end{array}$ \\
\hline Efficacy (\%) & & & & & 30.2 & 56.0 & 57.5 & 57.8 \\
\hline
\end{tabular}

Notes: Results are shown as mean \pm standard deviation for each rabbit group. Means were compared between ticks fed on vaccinated and control rabbits (merged) by one-way ANOVA followed by the Dunnett's t-test. In parentheses, \% of change in the corresponding parameter respect to the control: percentage of reduction for ingested blood, moulting, oviposition and fertility; increase for mortality

** $P<0.01$

Table 3 Effect of the vaccination with recombinant proteins CHI-GST, TSP1-GST + TSP2, RPP0, and PK4 administered in Montanide ISA 50 V2 on O. moubata specimens fed on control and vaccinated rabbits

\begin{tabular}{|c|c|c|c|c|c|c|c|c|}
\hline Parameter & $\begin{array}{l}\text { Developmental } \\
\text { stage }\end{array}$ & $\begin{array}{l}\text { Control } \\
\text { Merged }\end{array}$ & $\begin{array}{l}\text { Control } \\
\text { Montanide }\end{array}$ & Control SjGST & $\begin{array}{l}\text { CHI-GST (\% } \\
\text { reduction) }\end{array}$ & $\begin{array}{l}\text { TSP1- } \\
\text { GST + TSP2 (\% } \\
\text { reduction) }\end{array}$ & $\begin{array}{l}\text { RPPO (\% } \\
\text { reduction) }\end{array}$ & $\begin{array}{l}\text { PK4 (\% } \\
\text { reduction) }\end{array}$ \\
\hline \multirow[t]{3}{*}{$\begin{array}{l}\text { Ingested } \\
\text { blood (mg) }\end{array}$} & Males & $32.2 \pm 6.0$ & $32.1 \pm 5.1$ & $32.3 \pm 6.5$ & $29.4 \pm 0.4(8.4)$ & $30.9 \pm 1.1(3.8)$ & $\begin{array}{c}30.1 \pm 2.5 \\
(6.2)\end{array}$ & $\begin{array}{l}26.4 \pm 1.0 \\
(17.8)^{*}\end{array}$ \\
\hline & Females & $203.6 \pm 20.4$ & $204.9 \pm 20.7$ & $202.3 \pm 20.1$ & $\begin{array}{l}159.0 \pm 133.0 \\
(22.4)^{*}\end{array}$ & $\begin{array}{l}198.1 \pm 14.6 \\
(3.3)\end{array}$ & $\begin{array}{l}193.6 \pm 18.0 \\
(5.5)\end{array}$ & $\begin{array}{l}192.2 \pm 16.0 \\
(6.2)\end{array}$ \\
\hline & Nymphs-3 & $45.8 \pm 6.1$ & $45.8 \pm 4.5$ & $45.9 \pm 7.0$ & $41.5 \pm 2.9(9.3)$ & $44.1 \pm 1.2(3.6)$ & $\begin{array}{l}45.6 \pm 5.7 \\
(0.5)\end{array}$ & $\begin{array}{c}37.3 \pm 1.2 \\
(18.6)^{*}\end{array}$ \\
\hline \multirow[t]{3}{*}{ Mortality (\%) } & Males & $6.4 \pm 4.5$ & $5.9 \pm 7.1$ & $6.8 \pm 0.3$ & $6.7 \pm 7.3$ & $6.7 \pm 2.8$ & $6.0 \pm 8.5$ & $4.4 \pm 1.5$ \\
\hline & Females & $5.5 \pm 2.7$ & $6.6 \pm 0$ & $4.3 \pm 3.3$ & $6.3 \pm 0$ & $4.2 \pm 3.0$ & $2.1 \pm 3.0$ & $2.1 \pm 3.0$ \\
\hline & Nymphs-3 & $2.0 \pm 1.7$ & $2.0 \pm 2.0$ & $2.0 \pm 1.7$ & $0.7 \pm 1.0$ & $3.3 \pm 3.4(1.3)$ & $0.7 \pm 0.9$ & $1.9 \pm 1.6$ \\
\hline Moulting (\%) & Nymphs-3 & $67.2 \pm 1.7$ & $66.2 \pm 1.7$ & $68.2 \pm 1.3$ & $\begin{array}{l}63.4 \pm 36.5 \\
\quad(4.1)\end{array}$ & $61.8 \pm 8.4(6.6)$ & $72.0 \pm 4.0$ & $\begin{array}{l}57.4 \pm 3.6 \\
(13.3)^{*}\end{array}$ \\
\hline $\begin{array}{l}\text { Oviposition } \\
\text { (no. eggs/ } \\
\text { female) }\end{array}$ & Females & $197.3 \pm 18.8$ & $203.0 \pm 23.0$ & $191.0 \pm 12.0$ & $\begin{array}{c}158.0 \pm 2324 \\
(22.2)^{* *}\end{array}$ & $\begin{array}{l}182.0 \pm 14.0 \\
\quad(10.3)\end{array}$ & $\begin{array}{l}192.0 \pm 14.0 \\
(5.4)\end{array}$ & $191.0 \pm 6.7(5.9)$ \\
\hline $\begin{array}{c}\text { Fertility (no. } \\
\text { nymphs/ } \\
\text { female) }\end{array}$ & Females & $179.9 \pm 15.8$ & $181.0 \pm 21.1$ & $179.0 \pm 20.0$ & $\begin{array}{l}148.0 \pm 21.0 \\
(18.2)^{*}\end{array}$ & $\begin{array}{l}170.0 \pm 15.0 \\
\quad(6.0)\end{array}$ & $\begin{array}{l}179.0 \pm 20.0 \\
\quad(1.1)\end{array}$ & $180.0 \pm 7.0(0.5)$ \\
\hline Efficacy (\%) & & & & & 19.6 & 11.1 & 0 & 8.1 \\
\hline
\end{tabular}

Notes: Results are shown as mean \pm standard deviation for each rabbit group. Means were compared between ticks fed on vaccinated and control rabbits (treated with adjuvant alone) by one-way ANOVA followed by the Dunnett's t-test. In parentheses, \% of change in the corresponding parameter respect to the control: percentage of reduction for ingested blood, moulting, oviposition and fertility; increase for mortality

${ }^{*} P<0.05,{ }^{* *} P<0.01$ 
significant decreases in nymph moulting, female oviposition and female fertility. Accordingly, the vaccine efficacy of the OeCHI, OeTSPs, OeRPPO and OePK4 recombinant antigens against $O$. erraticus infestations was 30.2, $56.0,57.5$ and $57.8 \%$, respectively (Table 2 ).

On the O. moubata ticks, the recombinant antigens induced low protective effects, being mostly non-significant (Table 3). Only the anti-CHI response caused significant reductions in female feeding and reproduction; the anti-PK4 response caused significant reductions in nymph feeding and moulting, while the anti-TSPs response caused generalised non-significant reductions in tick feeding, nymph moulting and female reproduction. This resulted in lower global vaccine efficacies for the OeCHI, OeTSPs, OeRPPO and OePK4 recombinant antigens against $O$. moubata infestations: $19.6,11.1,0$ and $8.1 \%$, respectively.

Vaccine trial 2: humoral immune response and protective effects induced by jointly administered candidate antigens against soft tick infestations

The immune sera from all vaccinated rabbits showed IgG antibody titres higher than $1 / 12,800$ to each single recombinant antigen and around 1/3200 to the OeSEL synthetic peptide, confirming that all animals developed strong humoral responses.

Table 4 summarises the protective effects induced by the multicomponent vaccine against tick infestations. The anti-O. erraticus protective response was more intense than that of trial 1 , and mainly impacted female reproduction and nymph mortality, resulting in an increased global vaccine efficacy (66.3\%), which was $15 \%$ higher than the best protection reached with candidates tested individually. Regarding O. moubata, the vaccine cocktail induced similar but weaker effects than those in O. erraticus, namely, significant reductions in female reproduction and non-significant reductions in feeding performance, which resulted in a $25.6 \%$ vaccine efficacy, which was $30 \%$ higher that the best protection reached with individual candidates.

\section{Discussion}

Identifying highly protective antigens for tick vaccines development may be tackled by selecting protein candidates that play essential biological functions and share conserved sequence motifs to allow the simultaneous control of different tick species $[1,68]$.

In the present study, we focused on midgut chitinases, tetraspanins and RPP0 because these proteins are involved in important midgut physiological processes; several publications highlight their potential as vaccine candidates to control parasite infections [28, 29, 33, 42]. Additionally, and despite their unknown functions, we also focused on secreted proteins/peptides because of their accessibility to host antibodies ingested in blood, which make them the first-election candidate antigens for tick vaccine design $[69,70]$.

Topological analysis of the five selected candidates (chitinase, tetraspanins, RPP0 and secreted protein/ peptide) confirmed the transmembrane location and extracellular region/loops of OeCHI and OeTSPs (Additional file 2: Figure S1b and Additional file 3: Figure S2d, respectively), as well as the cytoplasmic location of OeRPP0, and the secreted nature of OePK4. Lineal B-cell

Table 4 Effect of the vaccination with a multiantigenic formulation containing candidate antigens OeTSP1, OeTSP2, OeRPP0, OePK4 and OeSEL in Montanide ISA 50 V2 on O. erraticus and O. moubata ticks fed on control and vaccinated rabbits

\begin{tabular}{|c|c|c|c|c|c|}
\hline \multirow[t]{2}{*}{ Parameter } & \multirow[t]{2}{*}{ Developmental stage } & \multicolumn{2}{|c|}{ Ornithodoros erraticus } & \multicolumn{2}{|c|}{ Ornithodoros moubata } \\
\hline & & Control & Vaccinated (\% reduction) & Control & Vaccinated (\% reduction) \\
\hline \multirow[t]{3}{*}{ Ingested blood (mg) } & Males & $3.1 \pm 0.3$ & $3.2 \pm 0.1$ & $26.2 \pm 2.2$ & $26.9 \pm 2.1$ \\
\hline & Females & $9.2 \pm 0.8$ & $9.3 \pm 1.1$ & $191.4 \pm 57.1$ & $180.1 \pm 12.9(5.9)$ \\
\hline & Nymphs-3 & $2.1 \pm 0.2$ & $2.0 \pm 0.2(4.8)$ & $5.5 \pm 0.7$ & $5.3 \pm 0.6(3.6)$ \\
\hline \multirow[t]{3}{*}{ Mortality (\%) } & Males & $2.8 \pm 5.3$ & $2.2 \pm 3.2$ & $6.1 \pm 2.6$ & $14.1 \pm 3.2(8.0)$ \\
\hline & Females & $1.1 \pm 2.7$ & $0 \pm 0$ & $9.0 \pm 5.6$ & $8.9 \pm 8.4$ \\
\hline & Nymphs-3 & $0.4 \pm 1.0$ & $13.2 \pm 7(12.8)^{* *}$ & $2.8 \pm 2.2$ & $3.3 \pm 1.0(0.5)$ \\
\hline Moulting (\%) & Nymphs-3 & $62.3 \pm 10.7$ & $61.8 \pm 9.0$ & $96.8 \pm 1.9$ & $94.4 \pm 4.8$ \\
\hline Oviposition (no. eggs/female) & Females & $62.2 \pm 7.3$ & $26.6 \pm 3.3(57.0)^{* *}$ & $200.0 \pm 21.0$ & $146.0 \pm 12.9(27.0)^{* *}$ \\
\hline Fertility (no. nymphs/female) & Females & $54.1 \pm 5.8$ & $21.5 \pm 2.7(60.2)^{* *}$ & $182.0 \pm 23.0$ & $137.5 \pm 11.0(24.5)^{* *}$ \\
\hline Efficacy (\%) & & & 66.3 & & 25.6 \\
\hline
\end{tabular}

Notes: Results are shown as the mean \pm standard deviation for each rabbit group. Means were compared between ticks fed on vaccinated and control rabbits by oneway ANOVA followed by the Dunnett's t-test. In parentheses, $\%$ of change in the corresponding parameter respect to the control: percentage of reduction for ingested blood, moulting, oviposition and fertility

** $P<0.01$ 
epitope predictions for the five candidates also verified the presence of this kind of epitope on the extracellular region/loops of OeCHI and OeTSPs (Figs. 1b, 2b) and throughout the amino acid sequence of OeRPP0 (Fig. 3b) and OePK4 (Fig. 4b) supporting their antigenicity. Interestingly, the 3D modelling of the candidates (except OePK4) showed that the predicted B-cell epitopes localize on the protein surface (Additional file 2: Figure S1c, Additional file 3: Figure S2e and Additional file 4: Figure S3b, respectively) where they could be easily reached by host antibodies. The multiple alignment of each candidate with its orthologues in other argasid and ixodid tick species showed that they share conserved structural and sequence motifs, including most of their antigenic extracellular regions (Additional file 2: Figure S1a, Additional file 3: Figure S2a, Additional file 4: Figure S3a and Additional file 5: Figure S4, respectively. This could facilitate the simultaneous targeting of different tick species if the candidates induce cross-protective immune responses.

The five candidates triggered robust immune responses in rabbits demonstrating high immunogenicity (Fig. 5a), which is in agreement with the linear B-cell epitope predictions and VaxiJen prediction of antigenicity for all of them except OeRPP0. These responses specifically recognised the inducing recombinant protein but did not cross-react with saliva of $O$. erraticus nor O. moubata (Fig. 5b). Consequently, the tick bites did not boost the vaccine-induced humoral responses (Fig. 5a), showing that natural tick-host contacts would not serve as boosting antigen doses in immunised hosts.

The immune sera to OeCHI and to OeTSPs recognised their respective native protein targets on the membrane protein extracts of $O$. erraticus ticks (Fig. 6b), confirming the presence of these proteins in the midgut proteome [23] and their predicted location to enterocyte membranes. The sequence identity shown by OeCHI and OeTSP1 to homologous proteins from O. moubata (Additional file 2: Figure S1a and Additional file 3: Figure S2a, respectively) can explain the fact that anti-OeCHI and anti-OeTSPs sera also recognised native chitinases and tetraspanins on the midgut protein extracts of $O$. moubata (Additional file 7: Figure S6), anticipating the possibility of some cross-species protection.

The anti-OeCHI immune response provided significant protection $(30.1 \%)$ to O. erraticus by reducing the moulting rate of nymphs without affecting other parameters (Table 2). This result is similar to the protective effect reported by [28] in mice vaccinated with a recombinant form of the $H$. longicornis chitinase CHT1, and strongly suggests that $\mathrm{OeCHI}$ is involved in moulting. Notably, the anti-OeCHI immune response also provided significant cross-protection to O. moubata (19.6\%), but it consisted of a general reduction in tick feeding performance, significant only in females, and significant reductions in female oviposition and fertility, without affecting nymph moulting (Table 3). These results resemble the phenotypic effect observed after RNAi gene knockdown of a salivary chitinase of $A$. americanum supposedly involved in tick feeding and cement cone stability maintenance [29]. Accordingly, it appears that the anti-OeCHI immune response recognises cross-reactive epitopes in an O. moubata chitinase that is likely involved in tick feeding and functionally distinct of the OeCHI identified in the present study. Based on the findings and data mining of this study, it seems that the tick GH-18 family has multiple members with different functions, and suggests that $\mathrm{OeCHI}$ might be a useful vaccine candidate antigen for the control of ornithodoros ticks.

Regarding tetraspanins, they are scaffold proteins that may participate in numerous and important intra- and inter-cellular biological processes due to their ability to interact with other proteins $[32,71]$. The anti-OeTSPs immune response provided significant protection (56\%) to $O$. erraticus and a notably lower cross-protection (11.1\%) to O. moubata, which is in agreement with the lower reactivity of the anti-OeTSPs sera to the $\mathrm{O}$. moubata midgut extracts (Fig. 6, Additional file 7: Figure S6). The protective effect was, however, qualitatively similar in both species: basically, reductions in nymph moulting and female oviposition and fertility (Tables 2, 3). These results suggest the involvement of OeTSP1 and/ or OeTPS2 in these processes, although further investigation is needed to determine the particular functions of these two tetraspanins. OeTSP1 and OeTSP2 only share $27.3 \%$ sequence identity to each other (Additional file 3: Figure S2c) and belong to different phylogenetic clades (Fig. 2a), suggesting that they may play different functions. Additionally, OeTSP1 is more conserved among tick tetraspanins than OeTSP2 (85-94.7\% and 30-58\%, respectively; Additional file 3: Figure S2a, b), suggesting that OeTSP1 could be the main cross-reactive antigen, and hence more useful for the simultaneous control of different tick species. Nevertheless, the present results show that these two tetraspanins, OeTSP1 and OeTPS2, can be suitable candidate antigens for vaccines aimed at the control of ornithodoros ticks, and establish the tick tetraspanin family as a source of potential vaccine targets for the first time.

The anti-OeRPP0 antibodies recognised the native form of the protein on the midgut membrane extracts of $O$. erraticus (Additional file 7: Figure S6) confirming its presence in the proteome [23] and its location as part of the ribosomes and the endoplasmic reticulum in the cytoplasm. As expected because of its high sequence identity (99.4\%, Additional file 4: Figure S3a), the anti-OeRPP0 also recognised the O. moubata RPP0 
orthologue on the membrane extracts of this species (Fig. 6d).

The anti-OeRPP0 response induced significant protection (57.5\%) to O. erraticus by reducing nymph moulting and female oviposition and fertility without any protective effect to O. moubata, despite the strong recognition of its RPP0 orthologue. This higher sensitivity of O. erraticus vs O. moubata to vaccines based on midgut antigens has already been observed and discussed by our team in previous studies, where it was reasoned that there may be particular factors in O. moubata (anatomical, physiological, molecular or microbial) that decrease the accessibility of immune effectors to their targets thus reducing the vaccine efficacy of the midgut concealed antigens against this species [20-22]. The protection provided by OeRPP0 to $O$. erraticus was lower than the $90-96 \%$ reached with peptide pP0 to ixodid ticks by [42, 43], which consisted in reduced feeding performance, reduced moulting and reproduction, and increased mortality. RPP0 is primarily involved in protein synthesis in ribosomes, so impairing this function might impact all the tick physiological processes dependent on the correct functioning of ribosomes. These would include the synthesis of salivary proteins and new cuticle that occur during feeding in ixodid but not argasid ticks $[72,73]$ and the increase in protein expression that take place during moulting and reproduction in both tick families. The higher number of physiological processes affected in ixodids would explain, at least partially, the higher impact in ixodids $v s$ argasids of the RPP0-based vaccines. However, RPP0 is a multifunctional protein, with a complex and incompletely understood biology $[44,45]$, which may complicate the explanation of how the host anti-RPP0 antibodies exercise their protective effects. Although further studies are needed to disclose which other functions of tick RPP0 are blocked by host antibodies and how the antibodies reach the target protein, the present results reinforce the notion that tick RPPO is a good broad spectrum candidate for tick vaccines, including argasids.

Regarding the selected "secreted peptide A0A293MVU8 (OePK4)", it grouped together with several PK-4 secreted proteins and secreted salivary gland peptides in the phylogenetic analysis. All of these proteins/peptides lacked functional annotations, except B7PVH8, which is annotated as "Cytochrome $c$ oxidase assembly protein", suggesting a functional association between OePK 4 and the mitochondrial respiratory chain (Fig. 4a). However, this association does not seem very probable since, far from being an integral component of mitochondrial inner membrane, OePK 4 is a soluble secreted protein, as indicated by its in silico analysis and confirmed by the fact that it is recognised by the antiOePK4 sera on the soluble fraction of midgut proteins in O. erraticus and O. moubata (Fig. 6b, c). Additionally, according to its description in the mialome (Additional file 1: Table S1), OePK4 would have a salivary origin and it would have reached the midgut with ingested saliva, where it would play hitherto unknown functions. This origin may however be controversial because the antiOePK4 antibodies did not reacted to saliva proteins and natural contacts with ticks did not increase the reactivity of anti-OePK4 sera (Fig. 5).

The anti-OePK4 response provided $57.8 \%$ significant protection to $O$. erraticus by reducing nymph moulting and female oviposition and fertility, and $8.1 \%$ crossprotection to $O$. moubata by reducing tick feeding, tick survival and nymph moulting. These results highlight the potential of OePK 4 as a protective candidate antigen and open the door to further studies to determine its function(s), tissue expression and expression regulation, and the mechanisms underlying its protective effects.

Finally, the results of trial 2 demonstrate that a combination of recombinant multi-epitope antigens targeting different tick physiological mechanisms increased vaccine efficacy compared with individual antigens. This highlights the potential usefulness and convenience of developing multicomponent vaccines for the control of ticks.

\section{Conclusions}

The recently obtained transcriptomic and proteomic data from the $O$. erraticus midgut has allowed us to apply a function-based approach to select candidate protective protein antigens from the tick midgut: one chitinase, two tetraspanins, the ribosomal protein P0 and one secreted protein PK4. The vaccination of rabbits with these candidates confirmed their predicted immunogenicity, since they all induced strong humoral immune responses. All candidates showed medium level protection against $O$. erraticus ticks, and all but RPP0 showed partial cross-protection against $O$. moubata. Protective effects were assumed to be the result of an antibody-mediated loss of function of the antigen targets. The results of the present study support that at least one chitinase and the ribosomal protein P0 from Ornithodoros ticks are promising protective antigens that might be included in vaccines aimed at control of multiple tick species. They also provide new protective antigens from argasids, namely, tetraspanins OeTSP1 and OeTSP2, and secreted protein PK4, that belong to protein families never tested before as protective antigens in ticks, which deserve further investigation. Finally, these results demonstrate that multicomponent vaccines increased vaccine efficacy compared with the individual antigens. New protective antigens from Ornithodoros spp. are still needed and will probably be 
identified by targeting tick proteins playing relevant biological functions for tick survival and pathogentick-host- interactions. Novel strategies for integrating multi-omics tools and data would facilitate a greater understanding of parasitic diseases. Proteogenomics approaches aimed at pathogen-tick-host-tick interactions will certainly allow abundant omics data to be acquired, the integration and analysis of which with modern functional studies will facilitate the identification of interesting targets and their valuation as vaccine candidate antigens.

\section{Supplementary information}

Supplementary information accompanies this paper at https://doi. org/10.1186/s13071-019-3768-1.

Additional file 1: Table S1. Transcriptomic data for chitinases, tetraspanins, ribosomal protein P0 and secreted proteins/peptides from the Ornithodoros erraticus midgut.

Additional file 2: Figure S1. Tick chitinases sequence alignment and $\mathrm{OeCH}$ topology prediction.

Additional file 3: Figure S2. Tick tetraspanins sequence alignment and OeTSPs topology prediction.

Additional file 4: Figure S3. Tick RPPO sequence alignment and OeRPPO topology prediction.

Additional file 5: Figure S4. Alignment of the amino acid sequences of A0A293MVU8_ORNER (OePK4) and orthologous peptides and proteins.

Additional file 6: Figure S5. Obtaining of candidate antigens as recombinant proteins.

Additional file 7: Figure S6. Analysis of reactivity of the vaccinated rabbit sera by ELISA.

\section{Abbreviations}

ABC: ATP-binding cassette transporter; ANOVA: analysis of variance; ASF: African swine fever; $\mathrm{CHI}$ : chitinase; FPKM: fragments per kilobase of transcript per million mapped reads; GH18: glycoside hydrolase-18; GPI: glycosylphosphatidylinositol; GST: glutathione S-transferase; LC-MS/MS: liquid chromatography-tandem mass spectrometry; NCBInr: National Center for Biotechnology Information non redundant; PBS: phosphate-buffered saline; PK4: secreted protein PK-4; RNAi: RNA interference; RPP0: 60S acidic ribosomal protein P0; RT-PCR: reverse transcription-polymerase chain reaction; SDS-PAGE: sodium dodecyl sulphate polyacrylamide gel electrophoresis; SEL: selenoprotein; SjGST: Schistosoma bovis glutathione S-transferase; TBRF: tick-borne relapsing fever; TPBS: PBS supplemented with 0.05\% Tween 20; TSP: tetraspanin.

\section{Acknowledgements}

The authors acknowledge the skilful technical assistance by Rocío VizcaínoMarín and María González-Sánchez from the Instituto de Recursos Naturales y Agrobiología de Salamanca (IRNASA, CSIC) (Spain). The authors also acknowledge support of the publication fee by the CSIC Open Access Publication Support Initiative through its Unit of Information Resources for Research (URICI).

\section{Authors' contributions}

RPS and AO conceived the study. RPS, RPM, POM and AO performed the experiments. RPS, RPM and AO performed data analyses. RPS wrote the draft, and other co-authors made additional suggestions. All authors read and approved the final manuscript.

\section{Funding}

This work was supported by project AGL2013-42745-P, funded by a grant from the Spanish Ministry of Economy and Competitiveness.

\section{Availability of data and materials}

The data supporting the conclusions of this article are provided within the article and its additional files. Raw data are available from the corresponding author upon reasonable request. The midgut transcriptome data used during this study were deposited in DDBJ/ENA/GenBank under accession number GFWV00000000 as a Transcriptome Shotgun Assembly project (BioProject: PRJNA401392).

\section{Ethics approval and consent to participate}

Animal experimentation in this study was performed according to the regulations established by the Ethical and Animal Welfare Committee of the IRNASA, CSIC, Spain, and the corresponding EU Law (Directive 2010/63/EU).

\section{Consent for publication}

Not applicable.

\section{Competing interests}

The authors declare that they have no competing interests.

\section{Author details}

${ }^{1}$ Parasitología Animal, Instituto de Recursos Naturales y Agrobiología de Salamanca (IRNASA, CSIC), Cordel de Merinas, 40-52, 37008 Salamanca, Spain. 2 Present Address: Proteomics Unit, Cancer Research Centre (IBMCC/CSIC) USAL/IBSAL), 37007 Salamanca, Spain.

Received: 16 July 2019 Accepted: 23 October 2019

Published online: 30 October 2019

\section{References}

1. de la Fuente J, Kopacek P, Lew-Tabor A, Maritz-Olivier C. Strategies for new and improved vaccines against ticks and tick-borne diseases. Parasite Immunol. 2016;38:754-69.

2. Boinas F, Ribeiro R, Madeira S, Palma M, de Carvalho IL, Núncio S, Wilson AJ. The medical and veterinary role of Ornithodoros erraticus complex ticks (Acari: Ixodida) on the Iberian Peninsula. J Vector Ecol. 2014;39:238-48.

3. Arias M, Jurado C, Gallardo C, Fernández-Pinero J, Sánchez-Vizcaíno JM. Gaps in African swine fever: analysis and priorities. Transbound Emerg Dis. 2017;65:235-47.

4. Talagrand-Reboul E, Boyer PH, Bergström S, Vial L, Boulanger N. Relapsing fevers: neglected tick-borne diseases. Front Cell Infect Microbiol. 2018;8:98.

5. Masoumi H, Goya MM, Vatandoost H, Zahraei SM, Mafi A, Asmar M, Piazak $\mathrm{N}$, Aghighi Z. The epidemiology of tick-borne relapsing fever in Iran during 1997-2006. Travel Med Infect Dis. 2009;7:160-4.

6. Chen Z, Yang X, Bu F, Yang X, Yang X, Liu J. Ticks (Acari: Ixodoidea: Argasidae, Ixodidae) of China. Exp Appl Acarol. 2010;51:393-404.

7. Schorderet-Weber S, Noack S, Selzer PM, Kaminsky R. Blocking transmission of vector-borne diseases. Int J Parasitol Drugs Drug Resist. 2017;7:90-109.

8. EFSA. Scientific opinion on African swine fever. EFSA J. 2014;12:3628.

9. EFSA. African swine fever. EFSA J. 2015;13:4163.

10. FAO. African swine fever threatens People's Republic of China. A rapid risk assessment of ASF introduction. Animal Health Risk Analysis. 2018. Assessment No. 5. Pome: FAO; 2018. p. 1-20.

11. Jurado C, Martínez-Avilés M, Torre AL, Štukelj M, Ferreira HCC, Cerioli M, et al. Relevant measures to prevent the spread of African swine fever in the European Union domestic pig sector. Front Vet Sci. 2018;5:77.

12. Díaz-Martín V, Manzano-Román R, Obolo-Mvoulouga P, Oleaga A, PérezSánchez R. Development of vaccines against Ornithodoros soft ticks: an update. Ticks Tick Borne Dis. 2015;6:211-20.

13. de la Fuente J, Contreras M. Tick vaccines: current status and future directions. Expert Rev Vaccines. 2015;14:1367-76.

14. Šmit R, Postma MJ. Vaccines for tick-borne diseases and cost-effectiveness of vaccination: a public health challenge to reduce the diseases' burden. Expert Rev Vaccines. 2016:15:5-7.

15. de la Fuente J. Controlling ticks and tick-borne diseases looking forward. Ticks Tick Borne Dis. 2018;9:1354-7. 
16. Kocan KM, De La Fuente J, Blouin EF, Garcia-Garcia JC. Anaplasma marginale (Rickettsiales: Anaplasmataceae): recent advances in defining host-pathogen adaptations of a tick-borne rickettsia. Parasitology. 2004;129:S285-300

17. Sojka D, Franta Z, Horn M, Caffrey CR, Mareš M, Kopáček P. New insights into the machinery of blood digestion by ticks. Trends Parasitol. 2013;29:276-85.

18. Sojka D, Pytelkova J, Perner J, Horn M, Konvickova J, Schrenkova J, et al. Multi-enzyme degradation of host serum albumin in ticks. Ticks Tick Borne Dis. 2016;7:604-13.

19. Araujo RN, Silva NCS, Mendes-Sousa A, Paim R, Costa GCA, Dias LR, et al. RNA-seq analysis of the salivary glands and midgut of the Argasid tick Ornithodoros rostratus. Sci Rep. 2019;9:6764.

20. Manzano-Román R, Encinas-Grandes A, Pérez-Sánchez R. Antigens from the midgut membranes of Ornithodoros erraticus induce lethal anti-tick immune responses in pigs and mice. Vet Parasitol. 2006;135:65-79.

21. Manzano-Román R, Garcia-Varas S, Encinas-Grandes A, Pérez-Sánchez R. Purification and characterization of a $45-\mathrm{kDa}$ concealed antigen from the midgut membranes of Ornithodoros erraticus that induces lethal anti-tick immune responses in pigs. Vet Parasitol. 2007;145:314-25.

22. Obolo-Mvoulouga P, Oleaga A, Manzano-Román R, Pérez-Sánchez R. Evaluation of the protective efficacy of Ornithodoros moubata midgut membrane antigens selected using omics and in silico prediction algorithms. Tick Tick Borne Dis. 2018;9:1158-72.

23. Oleaga A, Obolo-Mvoulouga P, Manzano-Román R, Pérez-Sánchez R. Midgut proteome of an argasid tick, Ornithodoros erraticus: a comparison between unfed and engorged females. Parasites Vectors. 2015;8:525.

24. Oleaga A, Obolo-Mvoulouga P, Manzano-Román R, Pérez-Sánchez R. De novo assembly and analysis of midgut transcriptome of the argasid tick Ornithodoros erraticus and identification of genes differentially expressed after blood feeding. Ticks Tick Borne Dis. 2018;9:1537-54.

25. Pérez-Sánchez R, Manzano-Román R, Obolo-Mvoulouga P, Oleaga A. Function-guided selection of midgut antigens from Ornithodoros erraticus ticks and evaluation of their protective efficacy in rabbits. Vet Parasitol. 2019;272:1-12.

26. Merzendorfer $\mathrm{H}$, Zimoch L. Chitin metabolism in insects: structure, function and regulation of chitin synthases and chitinases. J Exp Biol. 2003;206:4393-412.

27. Merzendorfer H. Insect-derived chitinases. Adv Biochem Eng Biotechnol. 2013;136:19-50.

28. You M, Fujisaki K. Vaccination effects of recombinant chitinase protein from the hard tick Haemaphysalis Iongicornis (Acari: Ixodidae). J Vet Med Sci. 2009;71:709-12.

29. Kim TK, Curran J, Mulenga A. Dual silencing of long and short Amblyomma americanum acidic chitinase forms weakens the tick cement cone stability. J Exp Biol. 2014;217:3493-503.

30. Oleaga A, Obolo-Mvoulouga P, Manzano-Román R, Pérez-Sánchez R. Functional annotation and analysis of the Ornithodoros moubata midgut genes differentially expressed after blood feeding. Ticks Tick Borne Dis. 2017;8:693-708

31. Assenga SP, You M, Shy CH, Yamagishi J, Sakaguchi T, Zhou J, et al. The use of a recombinant baculovirus expressing a chitinase from the hard tick Haemaphysalis longicornis and its potential application as a bioacaricide for tick control. Parasitol Res. 2006;98:111-8.

32. Levy S, Shoham T. Protein-protein interactions in the tetraspanin web. Physiology. 2005;20:218-24.

33. Moribe H, Mekada E. Tetraspanins in lower eukaryotes. In: Berditchevski F, Rubinstein E, editors. Tetraspanins, proteins and cell regulation. Dordrecht: Springer Science + Business Media; 2013. p. 187-201.

34. Murungi EK, Kariithi HM, Adunga V, Obonyo M, Christoffels A. Evolution and structural analyses of Glossina morsitans (Diptera; Glossinidae) tetraspanins. Insects. 2014;5:885-908.

35. Silvie O, Greco C, Franetich JF, Dubart-Kupperschmitt A, Hannoun L, van Gemert GJ, et al. Expression of human CD81 differently affects host cell susceptibility to malaria sporozoites depending on the Plasmodium species. Cell Microbiol. 2006;8:1134-46.

36. Tham TN, Gouin E, Rubinstein E, Boucheix C, Cossart P, Pizarro-Cerda J. Tetraspanin CD81 is required for Listeria monocytogenes invasion. Infect Immun. 2010;78:204-9.

37. Luo S, He M, Cao Y, Xia Y. The tetraspanin gene MaPIs1 contributes to virulence by affecting germination, appressorial function and enzymes for cuticle degradation in the entomopathogenic fungus, Metarhizium acridum. Environ Microbiol. 2013;15:2966-79.

38. Yang CF, Tu CH, Lo YP, Cheng CC, Chen WJ. Involvement of Tetraspanin C189 in cell-to-cell spreading of the dengue virus in C6/36 Cells. PLoS Negl Trop Dis. 2015;9:e0003885.

39. Vora A, Zhou W, Londono-Renteria B, Woodson M, Sherman MB, Colpitts TM, et al. Arthropod EVs mediate dengue virus transmission through interaction with a tetraspanin domain containing glycoprotein Tsp29Fb. Proc Natl Acad Sci USA. 2018;115:E6604-13.

40. Tebeje BM, Harvie M, Hong You H, Loukas A, McManus DP. Schistosomiasis vaccines: where do we stand? Parasites Vectors. 2016;9:528.

41. Gong H, Liao M, Zhou J, Hatta T, Huang P, Zhang G, et al. Gene silencing of ribosomal protein $\mathrm{PO}$ is lethal to the tick Haemaphysalis longicornis. Vet Parasitol. 2008;151:268-78.

42. Rodríguez-Mallon A, Fernández E, Encinosa PE, Bello Y, Méndez-Pérez L, Cepero $L$, et al. A novel tick antigen shows high vaccine efficacy against the dog tick, Rhipicephalus sanguineus. Vaccine. 2012;30:1782-9.

43. Rodríguez-Mallon A, Encinosa PE, Méndez-Pérez L, Bello Y, RodríguezFernández R, Garay H, et al. High efficacy of a 20 amino acid peptide of the acidic ribosomal protein P0 against the cattle tick, Rhipicephalus microplus. Ticks Tick Borne Dis. 2015;6:530-7.

44. Kar B, Mohapatra A, Mohanty J, Sahoo PK. Evaluation of ribosomal P0 peptide as a vaccine candidate against Argulus siamensis in Labeo rohita. Open Life Sci. 2017;12:99-108.

45. Singh S, Sehgal A, Waghmare S, Chakraborty T, Goswami A, Sharma S. Surface expression of the conserved ribosomal protein $\mathrm{PO}$ on parasite and other cells. Mol Biochem Parasitol. 2002;119:121-4.

46. Díaz-Martín V, Manzano-Román R, Valero L, Oleaga A, Encinas-Grandes A, Pérez-Sánchez R. An insight into the proteome of the saliva of the argasid tick Ornithodoros moubata reveals important differences in saliva protein composition between the sexes. J Proteomics. 2013;80:216-35.

47. Doytchinova IA, Flower DR. Identifying candidate subunit vaccines using an alignment-independent method based on principal amino acid properties. Vaccine. 2007;25:856-66.

48. Doytchinova IA, Flower DR. VaxiJen: a server for prediction of protective antigens, tumour antigens and subunit vaccines. BMC Bioinform. 2007;8:4.

49. Flower DR, Macdonald IK, Ramakrishnan K, Davies MN, Doytchinova IA. Computer aided selection of candidate vaccine antigens. Immunome Res. 2010;6(Suppl. 2):S1.

50. Tamura K, Stecher G, Peterson D, Filipski A, Kumar S. MEGA6: molecular evolutionary genetics analysis version 6.0. Mol Biol Evol. 2013;30:2725-9.

51. Krogh A, Larsson B, von Heijne G, Sonnhammer EL. Predicting transmembrane protein topology with a hidden Markov model: application to complete genomes. J Mol Biol. 2001;305:567-80,

52. Moller S, Croning MD, Apweiler R. Evaluation of methods for the prediction of membrane spanning regions. Bioinformatics. 2001;17:646-53.

53. Bendtsen JD, Jensen LJ, Blom N, von Heijne G, Brunak S. Feature based prediction of non-classical and leaderless protein secretion. Protein Eng Des Sel. 2004;17:349-56.

54. Fankhauser N, Maser P. Identification of GPI anchor attachment signals by a Kohonen self-organising map. Bioinformatics. 2005;21:1846-52.

55. Kelley LA, Mezulis S, Yates CM, Wass MN, Sternberg MJE. The Phyre2 web portal for protein modelling, prediction and analysis. Nat Protoc. 2015;10:845-58.

56. DeLano WL. The PyMOL molecular graphics system. San Carlos: DeLano Scientific; 2002.

57. Saha S, Raghava GPS. Prediction of continuous B-cell epitopes in an antigen using recurrent neural network. Proteins. 2006;65:40-8.

58. Saha S, Raghava GPS. BcePred: prediction of continuous B-cell epitopes in antigenic sequences using physicochemical properties, In: Nicosia G, Cutello V, Bentley PJ, Timmis J, editors. artificial immune systems: third international conference, ICARIS 2004, Catania, Sicily, Italy, September 13-16, 2004. Proceedings. Berlin: Springer; 2004. p. 197-204.

59. Larsen JEP, Lund $O$, Nielsen M. Improved method for predicting linear B-cell epitopes. Immunome Res. 2006;2:2.

60. de la Torre-Escudero E, Manzano-Román R, Pérez-Sánchez R, Siles-Lucas $\mathrm{M}$, Oleaga A. Cloning and characterisation of a plasminogen-binding surface-associated enolase from Schistosoma bovis. Vet Parasitol. 2010;173:76-84. 
61. Díaz-Martín V, Manzano-Román R, Siles-Lucas M, Oleaga A, Pérez-Sánchez R. Cloning, characterization and diagnostic performance of the salivary lipocalina protein TSGP1 from Ornithodoros moubata. Vet Parasitol. 2011;178:163-72.

62. Harlow E, Lane D. Antibodies: a laboratory manual. New York: Cold Spring Harbor Laboratory; 1988. p. 511-51.

63. García-Varas S, Manzano-Román R, Fernández-Soto P, Encinas-Grandes A, Oleaga A, Pérez-Sánchez R. Purification and characterisation of a P-selectin-binding molecule from the salivary glands of Ornithodoros moubata that induces protective anti-tick immune responses in pigs. Int J Parasitol. 2010;40:313-26.

64. Canales M, Enríquez A, Ramos E, Cabrera D, Dandie H, Soto A, Falcón V, Rodríguez M, de la Fuente JF. Large-scale production in Pichia pastoris of the recombinant vaccine Gavac against cattle Tick. Vaccine. 1997;15:414-22.

65. Aguirre ADAR, García MV, Szabó MP, Barros JC, Andreotti R. Formula to evaluate efficacy of vaccines and systemic substances against three-host ticks. Int J Parasitol. 2015;45:357-9.

66. Contreras M, de la Fuente J. Control of Ixodes ricinus and Dermacentor reticulatus tick infestations in rabbits vaccinated with the Q38 Subolesin/ Akirin chimera. Vaccine. 2016;34:3010-3.

67. Rathore AS, Gupta RD. Chitinases from bacteria to human: properties, applications, and future perspectives. Enzyme Res. 2015;2015:791907.
68. Contreras M, de la Fuente J. Control of infestations by Ixodes ricinus tick larvae in rabbits vaccinated with aquaporin recombinant antigens. Vaccine. 2017;35:1323-8.

69. Rappuoli R, Bagnoli F. Vaccine design: innovative approaches and novel strategies. Norfolk: Caister Academic; 2011

70. Richards SA, Stutzer C, Bosman AM, Maritz-Olivier C. Transmembrane proteins - mining the cattle tick transcriptome. Ticks Tick Borne Dis. 2015;6:695-710.

71. Dahmane S, Rubinstein E, Milhiet PM. Viruses and tetraspanins: lessons from single molecule approaches. Viruses. 2014;6:1992-2011.

72. Akov S. Blood meal digestion in ticks. In: Obenchain FD, Galun R, editors. Physiology of ticks. Oxford: Pergamon Press; 1982. p. 197-211.

73. Oaks JF, McSwain JL, Bantle JA, Essenberg RC, Sauer JR. Putative new expression of genes in ixodid tick salivary gland development during feeding. J Parasitol. 1991;77:378-83.

\section{Publisher's Note}

Springer Nature remains neutral with regard to jurisdictional claims in published maps and institutional affiliations.
Ready to submit your research? Choose BMC and benefit from:

- fast, convenient online submission

- thorough peer review by experienced researchers in your field

- rapid publication on acceptance

- support for research data, including large and complex data types

- gold Open Access which fosters wider collaboration and increased citations

- maximum visibility for your research: over 100M website views per year

At BMC, research is always in progress.

Learn more biomedcentral.com/submissions 